\title{
A survey of IoT protocols and their security issues through the lens of a generic IoT stack
}

\author{
Jonathan Tournier ${ }^{\mathrm{a}, \mathrm{b}}$, François Lesueur ${ }^{\mathrm{b}}$, Frédéric Le Mouël ${ }^{\mathrm{b}}$, Laurent Guyon ${ }^{\mathrm{a}}$, \\ Hicham Ben-Hassine ${ }^{a}$ \\ ${ }^{a}$ AlgosSecure, 57 bd Vivier Merle, Lyon, France \\ ${ }^{b}$ Université de Lyon, INSA-Lyon, CITI, F-69621, Villeurbanne, France
}

\begin{abstract}
The Internet of things (IoT) is rapidly growing, and many security issues relate to its wireless technology. These security issues are challenging because IoT protocols are heterogeneous, suit different needs, and are used in different application domains. From this assessment, we identify the need to provide a homogeneous formalism applying to every IoT protocols. In this survey, we describe a generic approach with twofold challenges. The first challenge we tackle is the identification of common principles to define a generic approach to compare IoT protocol stack. We base the comparison on five different criteria: the range, the openness of the protocol, the interoperability, the topology and the security practices of these IoT protocols. The second challenge we consider is to find a generic way to describe fundamental IoT attacks regardless of the protocol used. This approach exposes similar attacks amongst different IoT protocols and is divided into three parts: attacks focusing on packets (passive and active cryptographic attacks), attacks focusing on the protocol (MITM, Flooding, Sybil, Spoofing, Wormhole attacks) and attacks focusing on the whole system (Sinkhole, Selective forwarding attacks). It also highlights which mechanisms are different between two protocols to make both of them vulnerable to an attack. Finally, we draw some lessons and perspectives from this transversal study.
\end{abstract}

Keywords: IoT security, IoT protocols, IoT attacks, Generic approach, IoT comparison

\section{Introduction}

The IoT $[1,2,3,4]$ refers to a network of physical objects (things) able to communicate (amongst themselves and with external entities) and to sense and interact with the real world. These things have different computing and sensorial capabilities, providing complex interactions with their environment or users. IoT is rapidly growing as the number of devices strongly increases and should reach several billion in 2020 [5]. Many applications have already been developed in various domains, such as energy management, traffic control, mobility and healthcare. These applications have different needs and constraints (scalability, coverage and energy) [6] that induce a global 
heterogeneity in IoT. For instance, many protocols have been created to satisfy different types of applications, and each manufacturer wants to the maximum market share for its proposed objects and protocols. The associated market pressure pushes manufacturers and vendors to produce their items as quickly as possible.

The early release of these objects involves fast development, relegating the security to future work. Nevertheless, these objects are deployed in the real world, sometimes in critical infrastructure (healthcare and industry) and in houses. The threat is heightened, because devices are not isolated but rather are connected to a local network or even the Internet: without proper segmentation, the compromise of one device can lead to compromise of the entire network.

\subsection{IoT applications and threats}

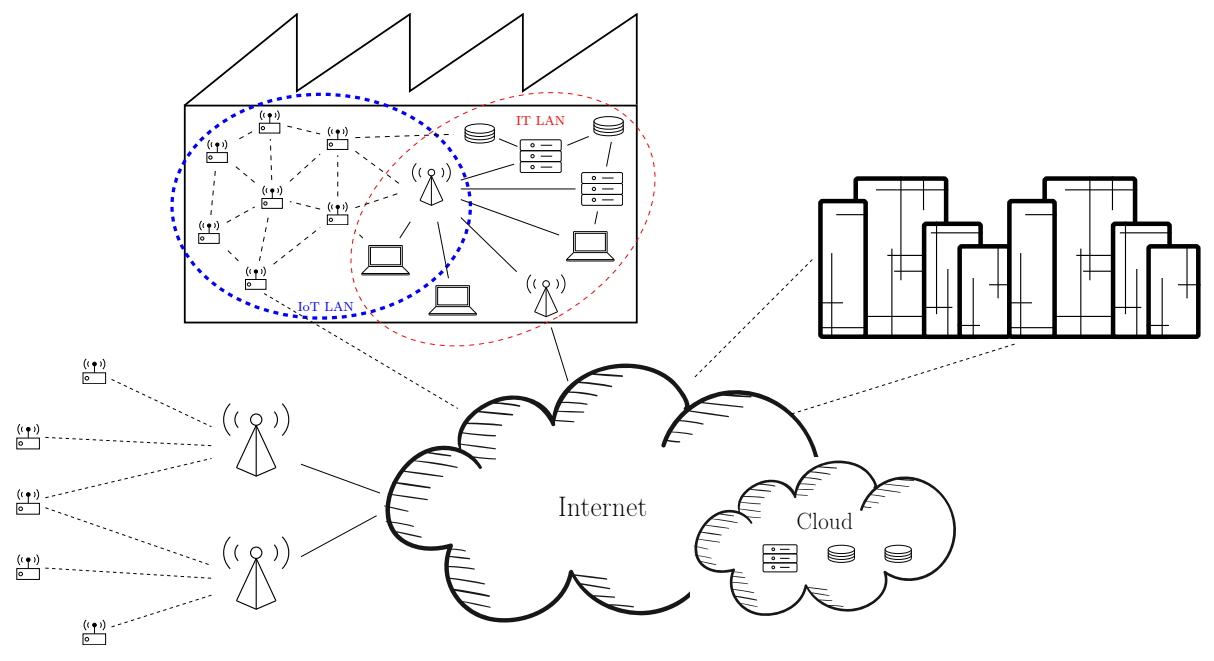

Figure 1: IoT eco-system representation.

IoT eco-system is heterogeneous with several subsystems, the whole interconnected through the Internet. Figure 1 is our vision of this IoT eco-system. We represent smart buildings, a smart factory and various isolated devices (considered as smart cities), in which all these actors may use cloud services and may be interconnected through Internet. For each actor in this eco-system, several IoT protocols and applications exist with specific requirements. For instance, the smart factory and smart buildings use a mix of short-range IoT protocols, such as Bluetooth low energy (BLE), ZigBee or WirelessHart. There may also be interconnected to a private network. These subsystems integrate actuator-sensor and monitoring applications. Smart cities use long-range protocols, such as LoRaWan, SigFox or NB-IoT. The use of cloud services is possible through an antenna acting as a gateway. Unlike smart buildings and smart factories, the devices of smart cities only communicate to the antenna and cannot use a peer-to-peer type of network. A large number of applications exist [7], such as video surveillance, traffic and parking managing and smart meters (whether, water, etc.). We can also integrate smart build and smart factories as a component of the smart city. 
With this vast amount of application comes several threats. Much related work shows that it is easy to take control of IoT devices and networks. For instance, many attacks $[8,9,10,11]$ allow an attacker to take full control of all devices in a network. In $[8,9]$, it was found that an attacker can control the door locks and the alarm of a smart home, whereas in [10], it was found that an attacker can take control of any Bluetoothenabled device. Once the attacker has control of the device, the attacker may retrieve sensitive data stored in the device, use it to spy the user or use it as a relay to spread the attack (Mirai malware [12] or its variants). An attacker may even inject a lethal dose of insulin through a connected pump [13], using classical vulnerabilities [14] such as man-in-the-middle (MITM) or buffer overflows. Multiple reports have also detailed how attackers can exploit IoT devices to retrieve the personal information of users $[15,16]$.

\subsection{Motivations}

The evolution of applications and communications in IoT has led to the development of multiple protocols, each of which attempts either to meet expectations of various uses or to target a specific domain. This plethora of protocols leads to multiple specifications and documentations, which may not be publicly available if the protocols are proprietary. Most related work on IoT security has engaged a specifically targeted protocol and the large number of (incompatible) protocols, leading to numerous (incompatible) security studies. However, even if protocols are incompatible and differ in characteristics and features, they inherit a similar architecture defining IoT systems. These protocols thus share abstract principles and are vulnerable to the same types of attacks. Because focusing $n$ times on $n$ different protocols is thus largely inefficient, we extracted in this survey these abstract features to be able to describe how any IoT protocol works and how all protocols are inherently vulnerable to the same set of generic attacks.

Moreover, because IoT is relatively young, its core protocols are evolving (new protocols or new versions). This leads to heterogeneous deployments, either today with different protocols on different parts of IoT or tomorrow with legacy protocols used alongside newer protocols. Some protocols can be designed to be secure, with the implementation of all security standards, but the environment in which they are used, the number and type of devices deployed and the need for interoperability with other or older protocols lead to many risks that create security issues. Dealing with this heterogeneity requires us to create an abstract model of these IoT protocols to be able to design the overall system with homogeneous formalism.

In this survey, we propose a generic approach to (1) identify common principles of IoT protocols and thus create an abstract protocol stack and (2) describe attacks within this abstract model. Our purpose is thus to consolidate the large and disparate yet related work we identified on IoT protocols and security in a coherent structure.

\subsection{Metareview}

In this section, we analyse and compare our work to existing surveys related to IoT security. Related surveys can be divided into three groups: those focused on the security of a unique protocol, those focused on the security of a specific (OSI) layer and those that encompassed IoT systems in their entirety. 
The first group of surveys $[17,18,19,20,21]$ focused on the security of a unique protocol stack, such as Z-Wave, Zigbee, BLE, WirelessHart or an open IPv6 low power wireless personal area networks (6LoWPAN) stack. Thus, they did not allow the reader to compare these protocols or gain an abstract view of the fundamental characteristics of IoT protocols. In this survey, we construct this abstract view, which is a prerequisite to analysing the fundamental security vulnerabilities of current and future IoT protocols, especially in expected heterogeneous systems.

The second group of surveys $[22,23,24,25,26]$ tackled the problem of comparing protocols, but each focused on only one OSI layer, such as data link (MAC), network, transport or session. Thus, they did not study or allow the reader to understand the (security) interactions amongst the layers. We are convinced that IoT systems are complex with, for instance, network keys provisioned through the application layer and many other strong interactions throughout the stack. We thus aimed to provide full-stack understanding of IoT protocols by describing and analysing all layers.

Finally, the third group of surveys $[27,28,29,30]$ provided a global view of security and associated challenges in IoT. However, this work was built upon the vision of the IoT architecture presented by Zhao and Ge [31]. This vision was based on a representation of generic IoT systems with three layers: the perception layer, the network layer and the application layer. The perception layer gathers all environmental data, the network layer transmits and processes these data, the application layer makes the link between the final user and the needed data. However, in our opinion, this IoT model is so abstract that it is hard to fit the security problems of IoT protocols into it. Furthermore, in the perception layer, some confusion lies between technologies and protocols, such as for RFID, WSN, ZigBee or even blockchain in the same group [27]. In our survey, rather than abstraction at the global IoT system scale, we advocate intermediate abstraction at the protocol level, which allowed us to precisely describe and compare IoT protocols, as well as their security issues. 


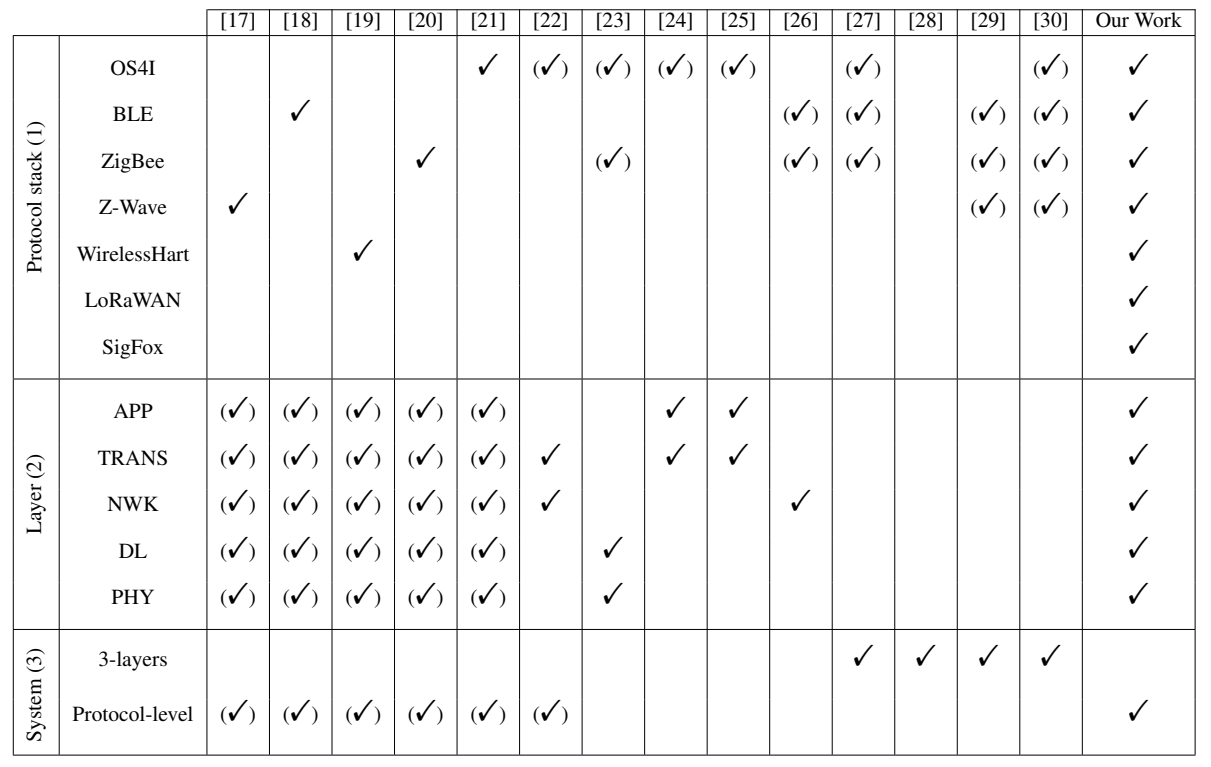

Table 1: Metareview summary according to three groups of surveys: (1) works focused on a unique IoT protocol stack, (2) works focused on a layer-level protocol comparison and (3) works focused on the security of the entire IoT system.

We summarise the meta-review section in Table 1. We represent the three groups we tackled in this paper. We then put a $\checkmark$ for each point addressed in a reference and a $(\checkmark)$ if the point is partially addressed. We leave an empty cell if the reference is not concerned by the point. For instance, we put a $\sqrt{ }$ in the row BLE (Protocol stack (1)) for the reference [18], because this work analysed the entire BLE stack, ranging from the physical (PHY) layer to the application layer. However, this work only focused on the BLE stack and did not compare or analyse other IoT protocols. Therefore, the second point, consisting of comparing multiple protocols for a specific layer (identified as Layer (2) in Table 1), is partially addressed, so we put a $(\checkmark$ ) in the corresponding cells. The last point (System (3)) concerns works focused on the security of IoT protocols with the vision of the entire system, whether it is represented in a 3-layer model presented by Zhao and Ge [31] (it corresponds to the 3-layer row in Table 1), or with an intermediate abstraction at the protocol level (corresponding to the row Protocol-level in Table 1). Because the work proposed in [18] addressed the security with a layer-level point of view, and not with a global vision of the BLE protocol, this work partially addressed this point, and the corresponding cell is filled with a $(\checkmark)$.

\subsection{Contribution and challenges}

As stated in Section 1.2, our contribution in this survey is to describe fundamental security issues of IoT networks, regardless of the IoT protocol stack used. These issues, as illustrated in Section 1.3, are presented in a large and disparate body of related work; 
consolidating this heterogeneous related work raises two challenges we tackle in this article :

- Identify a generic approach to comparing IoT protocol stacks: The same target of users and applications has several protocol stacks with their own designs and protocols and operating in different frequency bands with different ranges. Furthermore, documentations and specifications are specific to a protocol and have different levels of precision.

- Find a generic way to describe attacks regardless of protocols: Attacks on IoT systems are always described on a specific protocol. Describing them generically requires understanding of which ones are identical, which mechanism they depend on and whether they can be achieved on another protocol.

\subsection{Outline}

This survey gathered and analysed various works on security in IoT protocol stacks. In Section 2, we present our approach to generically compare various IoT protocol stacks. Section 3 details the most popular IoT protocols and fits them in our common frame. These two sections tackle the first challenge presented previously. Section 4 describes our approach to analysing the security of the parts of IoT systems, with Sections 5, 6 and 7 focusing on the security of packets, protocols and whole systems, respectively. Sections 4-7 thus correspond to the second challenge. We conclude this paper in Section 9 and discuss some perspectives.

\section{IoT protocol study}

\subsection{Comparison criteria}

In this section, we present the criteria we used to compare the IoT protocol stacks. We describe five criteria: range, openness, interoperability, topology (network architecture) and security practices. The four firsts are technical criteria, and the last one is a qualitative judgement.

\subsubsection{Range}

The first criterion used to classify the list of IoT protocol stacks is the area of action in which they can be used. We distinguished three types of network area: personal area network (PAN), local area network (LAN) and wide area network (WAN).

PAN protocols handle a limited number of devices on a short range. They are typically worn by the user and connect to a central point that must also be on the user. Typical setups encompass Bluetooth piconets with some wearable devices (watch or wristband) connected to a smartphone.

LAN protocols are defined by a limited number of devices within a restricted area, such as a house, factory or laboratory. However, they use a high debt rate in communications. The device range is about $100 \mathrm{~m}$. and the throughput is around $250 \mathrm{Kbps}$. Moreover, many of these protocols operate on the $2.4 \mathrm{GHz}$ industrial, scientific and medical (ISM) frequency band. 
WAN protocols are identified by a long range with low throughput and generally operate in a subgigahertz frequency band. The range can reach dozens of kilometres with a maximum throughput that ranges from 10 to $30 \mathrm{Kbps}$. Another characteristic is the regular use of cellular topology. However, the number of devices is not limited to an area. The more antennas, the more devices.

\subsubsection{Openness}

Our second criterion of classification is the level of transparency of the protocol. It corresponds to the availability and accessibility of information and resources of a protocol, such as technical specifications, implementations and source code. From this information, we split the degree of transparency into three levels: open (everything is published), half-open (not all resources are available) and closed (little information is available).

Open protocol stack. This level offers access to every part of the protocol stack. All protocols used in the stack are published; the overall stack is also published, and open implementations are available. This degree of transparency allows better comprehension of the protocol.

Half-open protocol stack. This level falls between an open protocol and a proprietary one. It offers only partial access to the protocol information. This means that it is possible to determine the protocols used in the stack but that one or many of them are proprietary. In another case, the company may use an open protocol but modify it without giving a detailed specification.

Closed protocol stack. This degree of transparency offers only the information the company wants to reveal. No specification is publicly available. Moreover, all attempts to reverse engineer the protocol without permissions can lead to legal actions.

\subsubsection{Interoperability}

The next criterion involves interoperability of the protocol stack with the existing world. Because Internet protocol (IP) is the standard interconnection, we determine whether it is possible for a device to be directly reachable, without proxy, from the Internet or from another IoT networks.

\subsubsection{Network architecture}

Network architecture (topology) represents the way devices organise themselves. IoT stacks use one or several types of architectures, with four main topologies. However, depending on the protocol stack, these topologies can be slightly modified to give different possibilities. Figure 2 represents the four categories of topologies we describe: star, tree, peer to peer (P2P) and cellular.

To provide a better generic vision of the available topology, we defined three types of nodes found in all protocols with different names or specific features: gateway, router and end device. The gateway is a node that controls the entire network and allows communication between the network and the Internet. The router forwards 

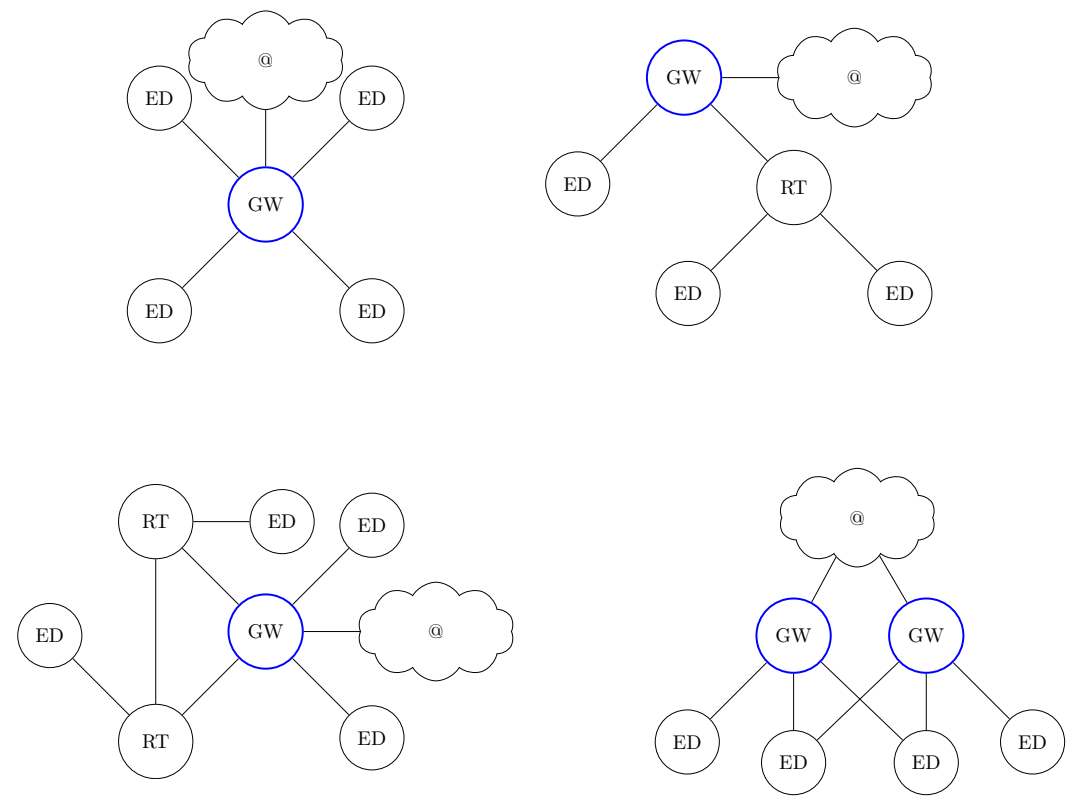

Figure 2: Types of topology: gateway (GW), router (RT) and end device (FD)

messages inside the network between devices. The end device is a node that can only send or receive messages.

The star topology is based on a master slave model, with a gateway (master) acting as a single central hub with which every end device (slave) can communicate.

The tree topology is based on parent child relationships, in which a router can be either a parent or a child and an end device is exclusively a leaf of the network. The gateway controlling the whole network is called the root node.

In the $P 2 P$ topology, there is no central hub that each communication must pass to reach the destination node. All routers can communicate with all other routers. When each router has a connection with all other routers, this topology is called a mesh.

Cellular topology, also called star of stars topology, is quite similar to star topology, with multiple gateways directly linked to the Internet. An important point in this topology is the possibility for a device to be connected to more than one gateway. Thus, all messages are sent several times, and the redundancy is handled in the Internet part of the topology.

\subsubsection{Security practices}

The security practice criterion is a personal judgement, ideally based on previous observations, regarding the attitude of the organisations supporting a protocol toward security issues. We focus primarily on responses to reported security vulnerabilities, because some protocols are open, have been well studied and can be improved by security researchers, whereas for others, the organisations behind them may be reluctant to collaborate with the security community. 
Regarding security issues, we consider communication, transparency or bug bounties to be good practices. In contrast, we consider organisations trying to limit security analysis and publication of their protocols to be undertaking bad security practices.

\subsection{Generic IoT stack}

To compare IoT protocol stacks in detail, we propose a common layered model as used in traditional networks with the OSI [32] or the transmission control protocol (TCP)/IP [33] models. Our proposed model is largely inspired by these wellestablished models but adapted to fit IoT protocol stacks. The OSI model (2), as shown in Figure 3, provides seven layers ranging from the physical layer to the application layer. Although the presentation layer and the session layer provide suitable functionalities for classic information technology (IT) networks, these two layers are not clearly defined and used in IoT systems. Thus, we decide to remove these layers from the generic IoT stack. The TCP/IP model (3), represented in Figure 3, provides a 4-layer stack, ranging from the network interface layer to the application layer. However, this model is used to determine how a device should be connected to the Internet. Therefore, the modification of protocols, such as TCP to UDP, with this model, may be complicated.

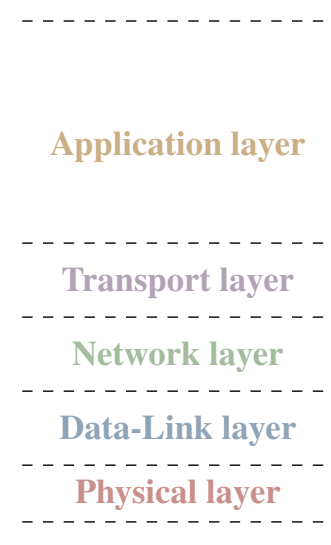

(1) Generic IoT stack

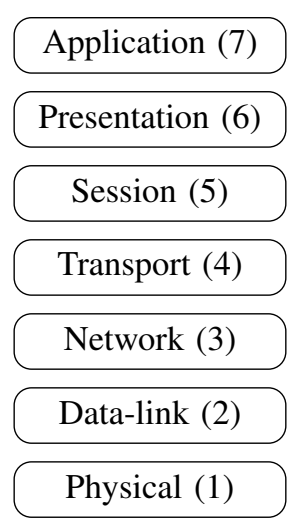

(2) OSI model

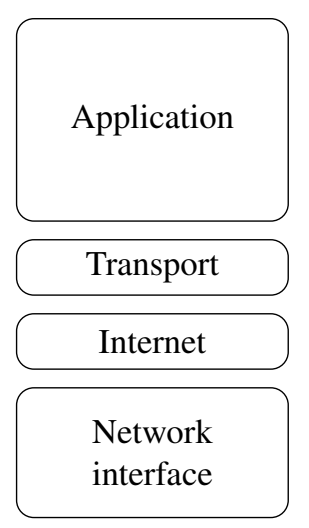

(3) TCP/IP model

Figure 3: (1) Generic IoT stack compared to (2) OSI model and (3) TCP/IP model.

The generic IoT stack (1) is represented in Figure 3. We use this model to compare all IoT protocol stacks presented in this survey. The model is composed of five layers, from the physical layer (PHY layer) to the application layer. Each layer provides specific features: the physical and data-link layers specify the radio frequency (RF) functionalities, the network layer defines routing and security capabilities and the transport and application layers specify the commands available in the protocol.

\section{IoT protocol stacks}

This section introduces IoT protocol stacks classified according to criteria defined in the previous section. For this survey, we chose to focus on recent protocols that 
are widely deployed, have been analysed by researchers or hackers and aim to propose security features: open stack for IoT (OS4I), BLE, Zigbee, Z-Wave, WirelessHart, LoRaWAN and Sigfox. We considered including narrowband IoT (NB-IoT) [34], but we concluded that its lack of current deployment, usage and security research made it irrelevant for a survey.

For each presented protocol stack, we first describe the stack structure, then present the associated routing mechanisms and finally analyse the security model. Each presented stack is labelled with the five criteria following this sequence:

\section{Range Openness Interoperability Types of topology Security practices}

All protocols presented in this section are summarised in Table 2, along with the criteria used to compare them and other features such as throughput, maximum number of nodes per network or security features.

\begin{tabular}{|c|c|c|c|c|c|c|c|c|}
\hline & \multicolumn{7}{|c|}{ Protocols } \\
\hline & & OS4I & BLE & ZigBee & Z-Wave & WirelessHart & LoRaWAN & SigFox \\
\hline \multirow{11}{*}{$\begin{array}{l}\text { 起 } \\
\text { 䓌 } \\
\text { L }\end{array}$} & Range & LAN $<100 m$ & LAN $<100 m$ & LAN $<100 m$ & LAN $<100 \mathrm{~m}$ & LAN $<100 m$ & WAN $\sim 5 \mathrm{~km}$ & WAN $\sim 10 \mathrm{~km}$ \\
\hline & Openness & Open & Half-open & Half-open & Close & Close & Close & Close \\
\hline & Interoperability & Yes & No & No & No & No & No & No \\
\hline & Topologies & Star, tree, mesh & Star, mesh & Star, tree, mesh & Mesh & Mesh & Cellular & Cellular \\
\hline & Security practices & Yes & Yes & Yes & No & No & No & No \\
\hline & Throughput & $250 \mathrm{Kbps}$ & $100 \mathrm{Mbps}$ & $250 \mathrm{Kbps}$ & $40 \mathrm{Kbps}$ & 250 Kbps & $50 \mathrm{Kbps}$ & $100 \mathrm{bps}$ \\
\hline & Frequency band & $2.4 \mathrm{GHz}$ & $2.4 \mathrm{GHz}$ & $2.4 \mathrm{GHz}$ & sub-GHz & $2.4 \mathrm{GHz}$ & sub-GHz & sub-GHz \\
\hline & Nodes max & Thousands & 32000 & 64000 & 232 & 30000 & $10^{4} / \mathrm{BS}$ & $10^{6} / \mathrm{BS}$ \\
\hline & Multi-hop & Yes & No/yes & Yes & Yes & Yes & No & No \\
\hline & Authentication & Yes & Yes/no & Yes & Yes & Yes & Yes & No \\
\hline & Encryption & AES-CCM & AES-CCM & AES-CCM & AES-CCM & AES-CCM & AES & No \\
\hline
\end{tabular}

Table 2: IoT protocol summary.

\subsection{OS4I}

In this section, we describe OS4I, a stack using protocols based on open technologies for each layer. This section provides more details than the others because this stack is the first presented and consists only of open (and thus documented) protocols. 


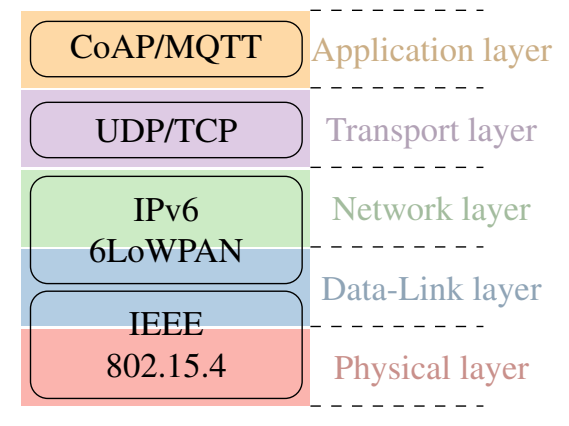

Figure 4: OS4I stack compared to the generic IoT stack.

\subsubsection{OS4I stack description}

Figure 4 depicts the IoT protocol stack only based on open technologies. The PHY layer and the data-link layer are defined by the Institute of Electrical and Electronics Engineers (IEEE) 802.15.4 standard [35]. An adaptative layer called 6LoWPAN appears between the data-link layer and the network one to ensure interoperability with non-IoT networks using IPv6. Various protocols can be used at the application layer such as constrained application protocol (CoAP) or message queue telemetry transport (MQTT). Depending on the IoT protocol deployed, the transport layer is defined by user datagram protocol (UDP) or TCP, respectively.

PHY layer. The PHY layer specifies the radio characteristics. This layer allows the protocol to operate in three possible frequency bands: $2.4 \mathrm{GHz}$ (with 16 channels), 915 $\mathrm{MHz}$ (with 10 channels) and $868 \mathrm{MHz}$ (with 1 channel). The throughput can be up to $250 \mathrm{Kbps}$ with a 10-m communication range. Moreover, the PHY layer offers collision avoidance and other features, providing real-time suitability.

Available frequency bands are split into two groups, the low band for 868 or 915 $\mathrm{MHz}$ (depending on the country in which the protocol is deployed) and the high band for $2.4 \mathrm{GHz}$. All groups are based on a direct sequence spread spectrum (DSSS), ensuring high resistance against interference. However, depending on the frequency band used, the modulation technique differs, implying different throughput. The low band offers $40 \mathrm{Kbps}$ using binary phase shift keying (BPSK) modulation, whereas the high band offers $250 \mathrm{Kbps}$ using offset quadrature phase shift keying (O-QPSK).

Medium access control (MAC) layer. The MAC layer provides functionalities like data transfer and channel scan. This layer also defines two types of nodes: reduced function device (RFD) and full function device (FFD).

An RFD can only act as a network end device (ED). These devices are generally equipped with sensors or actuators. Unlike the FFD, the RFD can only communicate with a single FFD. According to Figure 2, an RFD can only be an ED.

An FFD is more powerful and can act as an ED (as an RFD), an internal router relaying messages in a multihop network (router) or a network coordinator (personal area network coordinator (PAN coordinator)). The PAN coordinator can send beacons, 
providing synchronisation, communication and network join services. A single PAN coordinator per network controls the entire system and acts as a gateway linking the network devices to the Internet.

6LoWPAN protocol. One IoT challenges resides in the need to transpose classic IP networking to the world of low capability devices operating in the context of low power wireless personal area networks (LoWPAN). To achieve this, the Internet Engineering Task Force (IETF) specified the 6LoWPAN protocol [36, 37] in 2007. This protocol carries IPv6 packets over IEEE 802.15.4 networks. As a result, it allows interconnections between 6LoWPAN and classic IPv6 networks.

Using IPv6 allows 6LoWPAN to leverage its specific features, such as neighbour discovery, address resolution through link-local scoped multicast, duplicate address detection and router discovery. However, IPv6 packets are larger than those of IEEE 802.15.4. Therefore, the protocol offers various mechanisms to ensure a fit between the IPv6 datagrams and 6LoWPAN frame, despite eventual issues:

- Header compression: The adaptation layer optimises the space available for the data in an IPv6 packet by compressing its header. This means reducing the length of each header used within the packet, whether it is an IPv6, UDP or TCP header.

- Fragmentation and reassembly: Because the IPv6 maximum transmission unit (MTU) requirement does not match the size of the IEEE 802.15.4 frames, all IPv6 packets are fragmented into several segments and reassembled at the destination.

- Layer 2 forwarding: The adaptation layer allows the link layer to forward of IPv6 datagrams.

Header compression is defined in Request for Comments (RFC) 6282 [38] that updates the old RFC 4944 [39] to improve the methods used to compress IPv6 and UDP headers. Similar mechanisms exist [40] to perform header compression for TCP, but we did not address them in this paper and instead focused on UDP. The header compression level can vary according to the chosen type of communication amongst the three available options:

- Link-local unicast communication: This type of communication occurs between two devices within a 6LoWPAN network. The devices addresses are generated from the link-local prefix and the IEEE 802.15.4 addresses. In this context, both addresses can be determined from the IPv6 header. Thus, the IPv6 header can be compressed to 2 bytes.

- Multicast communication: This type of communication relates to interaction devices outside the link-local scope but still within the same network. Through use of the shared context for multicast, header compression can be reduced to 4 bytes.

- Global communication: Broader communication also occurs outside of the linklocal scope. However, global communication makes multiple IP hops and reaches a destination outside of the network. Hence, the source and destination addresses must be set in the header, increasing it up to 7 bytes. 
Transport layer. The transport layer relies on UDP or TCP. The choice between these two protocols is tied to the protocol used on the upper layer. There are pros and cons for each protocol. For instance, TCP allows better quality of service (QoS) with robust message delivery, whereas always-on TCP connections limit usage in a constrained environment. In a same way, UDP provides fast, efficient message transmission but lacks the reliability and service guarantee of TCP.

Application layer. The application layer is an abstraction layer that specifies the protocol used to ensure the communication between hosts. Such communication can be either between ED or between the server and and end device. Based on OS4I, shown in Figure 4, we describe two open protocols: CoAP built upon UDP and MQTT built on TCP.

The Constrained Restful Environments (CoRE) workgroup proposed CoAP [41, 42], a protocol designed for devices with limited resources (battery, memory or even computing power). This protocol is based on a representational state transfer (REST) architecture like hypertext transfer protocol (HTTP), but its complexity is reduced by the use of UDP rather than TCP. Moreover, methods such as GET, POST, PUT and DELETE are included in the protocol, allowing compatibility with HTTP. Thus, CoAP extends the unification between classic information technology (IT) and IoT defined by 6LoWPAN with the capability for well-known web applications to interact with IoT machine-to-machine (M2M) applications.

Furthermore, CoAP offers numerous functionalities [43], such as an asynchronous transaction model, an embedded web transfer protocol (coap://), lost packet retransmission and support of unicast and multicast communications. Furthermore, the use of UDP implies a new protocol that ensures the security of communication, namely, datagram transport layer security (DTLS) [44, 45, 46]. Finally, CoAP provides functionalities unavailable in HTTP, namely, observe, block and discovery:

- Observe: This feature is a mechanism of subscription to a resource. The client does not request the server to acquire a the fresh value; rather, the server notifies the client that the value has changed and sends it.

- Block: This feature can be compared with IP fragmentation. However, instead of relying on IP fragmentation that requires more resources, CoAP enables a pair of block options, allowing the transfer a large amount of information in multiple sequences of request response.

- Discovery: This feature allows a client to know which resources are available on the server or gives the client the location of each resource. CoAP introduces the new approach of web discovery service by including the uniform resource identifier (URI) /.well-known/core, in which each server provides a description of its resources. Thus, the client can send a GET request to discover services.

MQTT is an open standard protocol originally created by IBM. This protocol is based on a publish subscribe model designed for classic communications as well as lightweight M2M communications [47]. Contrary to CoAP, MQTT is built upon TCP, 
so the security of the protocol relies on the traditional secure sockets layer/transport layer security (SSL/TLS) protocol.

MQTT provides some interesting characteristics that make it compatible with constrained networks. It ensures a small amount of transport overhead and limits protocol exchanges to reduce network traffic in an environment with low bandwidth. The use of TCP with MQTT provides a solution to mitigate predictable connection losses in an unreliable network. Moreover, MQTT ensures QoS, which is split into three levels :

QoS O (at most once): This level depends on the reliability of the TCP/IP network. This is the lowest level of QoS, so the messages are sent only once and the recipient can receive or not receive the message. Moreover, the recipient does not send an acknowledgement (ACK) response.

QoS 1 (at least once): This level assures that messages will arrive, but duplication may occur. For each message received, the recipient must send an ACK response to confirm the receipt.

QoS 2 (once): This is the highest level of QoS and the messages are assured arrival with the recipient without duplication or loss. This exchange is performed by a four-way handshake mechanism between the broker and the client.

\subsubsection{Routing in 6LoWPAN}

Multihop routing allows transmission of packets amongst distant devices. This action can be done through multiple devices, commonly called transmission over multihop. The routing is important in networks such as 6LoWPAN, in which the capabilities of nodes are limited. The 6LoWPAN protocol is an adaptation layer inserted between the network layer (IPv6) and the data-link layer (IEEE 802.15.4 MAC).

Depending on the layer by which the routing process is done, we can split routing protocols into two categories: mesh-under and route-over [48, 49]. For the mesh-under scheme, the routing decision is taken on the adaptation layer based on the link layer, whereas the network layer controls the routing decision for a route-over scheme.

Mesh-under routing. The adaptation layer performs IPv6 fragmentation to forward IPv6 packets over the IEEE 802.15.4 radio link. In a mesh-under scheme, the packet forwarding is transparent to fragmentation. There is no control of the fragmentation header. Thus, only the information contained in the IEEE 802.15.4 header and the mesh addressing header is used to forward a packet or a fragment. The source and final destination are included in the mesh addressing header, and the address of the current and next hop are contained in the IEEE 802.15.4 header. In this way, it is possible to know whether the fragment or packet has reached the destination or must be forwarded to the next hop without reassembly of the packet. This is the difference between an IP hop and a simple hop. Contrary to the route-over scheme, in mesh-under routing, the IPv6 packet does not have to be reassembled at each hop. However, if a fragment is missing, then all fragments must be retransmitted. According to [49], it is possible 
to reduce the number of fragments that must be retransmitted by using the selective retransmission for the mesh-under scheme.

The IETF and the 6LoWPAN Working Group published several protocols to ensure compatibility with 6LoWPAN features. The first one was the ad hoc on demand distance vector routing algorithm (AODV) [50] in 2003; however, it was not adapted for the mesh-under scheme and link-layer addresses. The second one was LOAD [51] a derivative version of AODV, but its development was suspended. LOADng [52] was finally proposed to provide a better compatibility with mesh-under routing. It is also derived from the AODV routing protocol and aspires to become the standard for this type of routing.

Route-over routing. The other way to provide routing in a 6LoWPAN network is to forward data frames at the network layer. This routing scheme is based on IP routing, whereby each link-layer hop corresponds to an IP hop. The IPv6 hop-by-hop and IP routing table options are mainly used in route-over routing. The former option carries elective information that need to be verified by each hop on the delivery path. The latter option provides information that lets the current hop know which hop must receive all fragments. These two options imply that all fragments must be reassembled at each hop to retrieve IPv6 header information. Thus, when the adaptation layer receives all fragments, it creates an IP packet and sends it to the network layer. If the final destination matches with the current hop, then the packet is transmitted to the higher layer. However, if it does not match, then the IP packet comes back to the adaptation layer that fragments it and forwards it to the next hop based on routing table information.

As in mesh-under routing, if a fragment in the route-over scheme is missing during reassembly, all fragments must be retransmitted. The difference in the latter scheme is that the retransmission is made not from the source but rather from the last hop that forwarded the IP packet. According to [49], selective retransmission for the route-over scheme allows it to retransmit only lost fragments.

the routing over low power and lossy (ROLL) Working Group formed by the IETF proposed a routing protocol for low-power and lossy networks (RPL) in 2008 [53, 54, 55]. RPL is a distance vector IPv6 routing protocol for low-power and lossy networks and builds a destination oriented directed acyclic graph (DODAG) to represent the network as logical routing topology. Thus, it is easier to compute operations such as best path.

\subsubsection{Security in $O S 4 I$}

OS4I is a stack based on multiple IoT protocols, in which each defines a specific layer of the stack. Each layer is independent and provides its own security mechanisms.

Security in IEEE 802.15.4. We only consider the security provided by the IEEE 802.15.4 MAC layer. It offers data encryption using the AES-CCM* block cipher mode of operation. This protocol is a variation of the AES-CCM algorithm; it provides the same features and adds the possibility of using encryption-only capabilities. The size of the key is fixed to 128 bits, as is as the size of the plaintext block. 


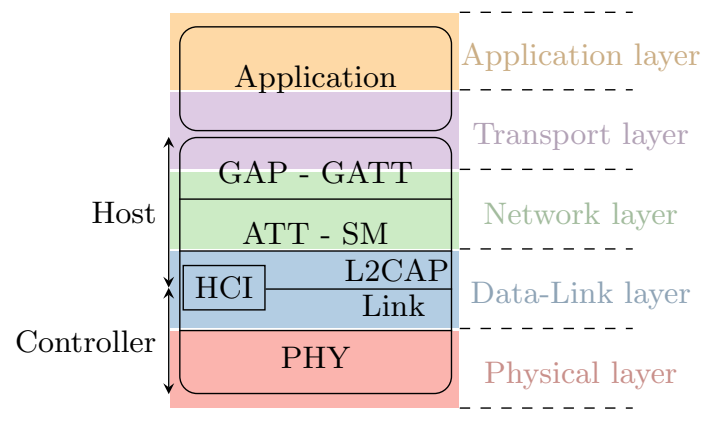

Figure 5: BLE stack. Host controller interface.

Security in network layer. The network layer relies on IPv6, so we only consider IP security (IPSec) as a security mechanism to ensure interoperability with the Internet. Raza et al. presented approaches of IPSec [56] and key exchanges [57] to make them suitable for constrained environments.

Security in the transport layer. The security provided by this layer depends on the protocol used at the application layer. If CoAP is used, then the transport protocol is UDP and the security mechanism provided is DTLS [44, 45, 46]; if MQTT is deployed, then TCP is used as the transport protocol and the corresponding security mechanism is SSL/TLS.

\section{2. $B L E$}

LAN, PAN Half-Open No Star, Mesh Yes

BLE is a protocol developed by the Bluetooth special interest group (SIG) and introduced in version 4.0 of the Bluetooth specification [58]. BLE is a wireless protocol for short-range communication whose field is similar to that of classic Bluetooth, such as control and monitoring applications. By design, BLE is suitable for low-power devices and meets the requirements for IoT.

There is no compatibility between devices implementing BLE and those implementing classic Bluetooth. Hence, many devices implement both protocols and are called dual-mode devices.

\subsubsection{BLE stack description}

Figure 5 represents the BLE stack compared with the generic IoT stack. The application layer is the only one that is not defined in the BLE specification. For the other layers, we can split them into two parts: the host and the controller.

The host handles the upper layers of the stack which are the logical link control and adaptation protocol (L2CAP), the attribute protocol (ATT), the security manager (SM), the generic access profile (GAP) and the generic attribute profile (GATT) protocol. The controller handles the lowest layers of the protocol, which are the link and PHY layers. The two parts can communicate to each other using the host controller interface (HCI). The following sections present an overview of each layer of the BLE stack [59]. 
PHY layer. Like most LAN wireless protocols presented earlier, BLE operates in the $2.4 \mathrm{GHz}$ ISM band with 40 channels and a space of $2 \mathrm{MHz}$. BLE distinguishes between two types of RF channels: advertising and data channels. Three advertising channels are used for device discovery, and 37 data channels are used for bidirectional communication between devices. An adaptive frequency hopping mechanism is used on data channels to reduce the effects of interference, fading, multipath, etc. This mechanism randomly chooses one of the 37 available data channels to communicate at a given time.

The PHY layer uses Gaussian frequency-shift keying (GFSK) modulation for all channels and provides a data rate of 1 Mbps.

Link layer. The link layer is responsible of creating and maintaining communications, as well as advertising and scanning. These functionalities are ensured by six roles paired defined at this layer:

- Advertiser-scanner: An advertiser device sends announcements through advertising channels to be discovered. A scanner device listens on these channels while waiting to discover a device.

- Slave-master: A slave device is an advertiser that accepts a connection. A master device is a scanner that has initiated a connection that is accepted.

- Broadcaster-observer: A broadcaster device sends announcements and denies all connections. An observer device only detects devices that make announcements without creating a connection.

These roles are defined according the type of communication used, whether it is unicast (P2P) or broadcast. In a unicast connection, two role pairs are involved; the advertiser-scanner and the slave-master roles. The broadcaster-observer role pair is only used in a broadcast connection.

Unicast connections follow various phases, during which the roles of the devices evolve. A P2P connection starts with a discovery phase involving two devices. The first device that wishes to be discovered takes on the role of advertiser, and the one that wishes to connect takes on the role of scanner. The advertiser device sends advertising packets claiming that it is a connectable device until a scanner device finds it. After filtering and analysing the information contained in advertising packet, the scanner device wishing to initiate a connection takes on the role of an initiator and sends a connection request (CONNECT_REQ) packet to the advertiser. This phase corresponds to the connecting phase. The next phase is known as the connected phase and begins when the advertiser accepts the CONNECT_REQ packet. During this phase, the advertiser becomes the slave and the scanner becomes the master.

A master can handle several connections with different slaves. Since the Bluetooth 4.1 specification, a slave is no longer required to connect with only one master and can be simultaneously connected to multiple masters. Nevertheless, regardless of the Bluetooth version used, a slave must respond to a master.

Sleep mode by default for slaves allows improved energy savings. Slaves are woken periodically to listen to packets from a master. The period is determined by the master. 
Moreover, the master handles all communications using a time division multiple access (TDMA) scheme. It also determines the use of the frequency hopping algorithm and shares all needed information with the slaves.

Each connection between master and slaves brings division of the physical channel into connection events, which are nonoverlapping time units. All connection events are initiated by the master and use the same data channel frequency throughout the event. A connection event is open until no more data are transmitted or an error occurs

L2CAP layer. The L2CAP protocol is a simplified version of classic Bluetooth L2CAP. The main goal of this protocol is to multiplex the data of the ATT and SM layers into the link layer. Contrary to classic Bluetooth L2CAP, the functionality of segmentation and reassembly is not necessary in BLE, because the higher layers can take care of their payload size.

ATT and SM layers. ATT is used atop an L2CAP channel to define communication between devices using a client server scheme, in which the server stores a set of data structures, called attributes, containing the information managed by GATT. A client can access attributes by requesting the server. Many types of operations are allowed, such as read and write attribute values; however, all transactions follow a strict stopand-wait scheme. This means that no other request can be sent until the response to the previous request has been received and processed.

The SM protocol is a complement of the security features defined in BLE. The SM protocol is used to take care of the three phases processed on devices' connection, in other words, the pairing session. A pairing session is determined by three phases; (1) announcement of the capabilities of each device, (2) generation of the short-term key (STK) and (3) distribution of the long-term key (LTK), the connection signatureresolving key (CSRK) and the identity-resolving key.

GAP and GATT layers. GATT is built atop ATT. It determines the client server roles regardless of master slave role. It also gives structure to attributes in the form of services and characteristics, in which a service is a set of characteristics and a characteristic is a set of attributes. Hence, GATT acts as a framework that uses ATT. The main roles of GATT are the discovery of services and the exchange of characteristics between devices.

GAP is a framework that specifies device roles, models and procedures to enable discovery amongst nodes, the broadcasting of data and the establishment of secure connections. Four roles can be adopted by a device to join a network: broadcaster, observer, central or peripheral. Broadcaster and observer roles are the same as the ones defined at the link layer. These roles are used to implement unidirectional and connectionless communications. The peripheral and central devices are involved in bidirectional and connection-oriented communications. The peripheral device relies on a slave role, and the central device relies on a master role. A procedure is a sequence of actions that a device must perform to accomplish its tasks. A model is the state in which a device must perform a procedure. Hence, a model depends on the role played by the device, and a procedure depends on the model and the role. 


\subsubsection{Routing in $B L E$}

Originally, BLE focused on a star topology, in which the device playing the master role is the central node and the slaves are the end points. Thus, all communications passed through the central node. BLE topology can also be called a master slave topology because of the role played by the devices. Piconet is another word to cover in networking in BLE and is not described further here.

With Bluetooth core specification 5.0 [60], mesh topology became available on BLE [61]. From this perspective, many mesh network mechanisms have emerged; [62] provides a taxonomy of the different BLE mesh networks.

According to [61], BLE mesh networking follows a message-oriented communication type using a publish subscribe messaging system. However, BLE adapts it with a relay principle to be more convenient in large spaces. Thus, a message can be retransmitted through many devices acting as relay with a limit of 127 hops. Finally, BLE uses a flooding approach to publish and relay messages. Thus, all devices in the range of the sender device receive the message, all devices acting as a relay retransmit it to all devices in their range and so on.

\subsubsection{Security in $B L E$}

The security in BLE comprises multiple security modes with multiple security levels. The security mode and the security level are based on the method of pairing that is used between the devices.

Pairing. Pairing implies the authentication of two devices with the use of a shared secret. This authentication allows the encryption of the link with STK and the distribution of LTK to ensure encrypted communication. Four procedures generate an STK during a pairing:

- Just works: Use a default pin code.

- Passkey: Display a pin code on a device that must be used by the other one.

- OOB: Use a side channel to transmit data (NFC, QRcode, etc.).

- Numeric comparison: Display pin codes on both devices; verification must be done by the user.

Security in BLE is split into two modes, with multiple security levels for each mode:

Security mode 1. This mode provides security based on encryption with four security levels:

- Level 1: No security (no authentication and no encryption)

- Level 2: Unauthenticated pairing with encryption

- Level 3: Authenticated pairing with encryption

- Level 4: Authenticated LE Secure Connections pairing with encryption 


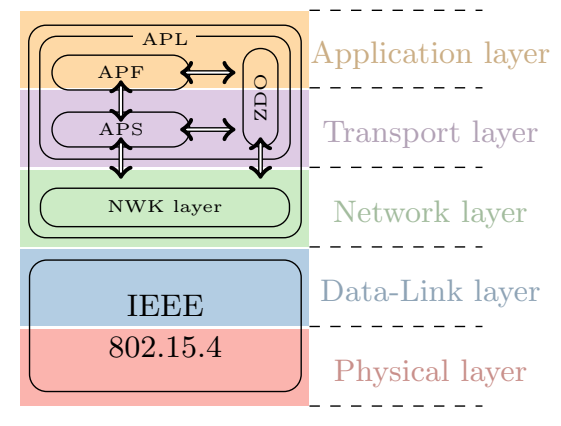

Figure 6: Detail of the Zigbee protocol.

Security mode 2. This mode enforces security based on data signing and provides two security levels:

- Level 1: Unauthenticated pairing with data signing

- Level 2: Authenticated pairing with data signing

The encryption is based on the 128 bit AES-CCM protocol and the shared secret key is generated using LTK. The authentication is provided by using CSRK.

\subsection{Zigbee LAN Half-Open No Star, Mesh, Tree Yes}

Zigbee is an IEEE 802.15.4-based protocol specified by the Zigbee Alliance.

\subsubsection{Zigbee stack description}

The Zigbee protocol is based on the IEEE 802.15.4 standard for the physical layer and the data-link layer (presented in Section 3.1.1). The standard then specifies the upper layers, as shown in Figure 6.

According to [20], these upper layers are composed of the network layer and an application layer, with the application layer consisting of an application framework (APF), Zigbee device object (ZDO) and application sublayer (APS). The following sections detail each part of these layers.

network (NWK) layer. The network layer provides numerous functionalities, amongst which are multihop routing, route discovery and maintenance, security and joining or leaving network.

Application Framework (APF). APF is composed of 254 application object (APO), in which each APO is a piece of software that controls a specific hardware unit (switch, lamp, etc.) available on the device. All APOs can communicate and interact amongst themselves, with a locally unique endpoint number assigned to each APO.

Zigbee Device Object (ZDO). ZDO offers services to the APOs and allows them to discover devices and the services they implement in the network. It also provides such services as communication, network and security management services. 
Application Sub Layer (APS). APS links APO and ZDO to manage data transfer services between them. To create an application, APS must conform to an existing profile provided by the Zigbee Alliance. The message format and protocol for interactions between APO are defined by the application profile. The main advantage of the application profile is interoperability amongst applications developed by different manufacturers.

\subsubsection{Routing in Zigbee}

Zigbee is based on the IEEE 802.15.4 standard to specify the PHY and MAC layers. Thereby, Zigbee provides star, tree and mesh topologies. Moreover, each device that composes a Zigbee network is either an FFD or an RFD, but their names change to become Zigbee ED, Zigbee router, accompanied by a Zigbee coordinator. A Zigbee ED is an RFD or an FFD acting as a simple device. A Zigbee router is an FFD with routing capabilities. A Zigbee coordinator is unique to the Zigbee network and corresponds to an FFD controlling the entire network.

The routing algorithm used in Zigbee depends on the network topology used.

Tree topology. The only possible algorithm within a tree topology consists of basic parent child links established as a result of join operations (called tree-based routing). Routers maintain a list of their address and the address information associated with their children and parents. Communications work with a beaconing system; the parent and the child who want to communicate must synchronise themselves before the start of communication.

Mesh topology. Although more complex to handle and with beaconing disallowed, a mesh type of network is more robust than a tree topology and resilient to faults. Routers maintain a routing table and employ a route discovery algorithm to construct or update these data structures on the path nodes. The routing table is consulted only if the trivial routing for the next hop to the destination is not possible. If the destination is not found, even in the routing table, then the discovery routing algorithm is started. In the worst case, if the resources needed for the discovery are unavailable, then routing falls back to a tree-based mode.

Route discovery algorithm. A route discovery algorithm is a necessary process to create a routing table entry in the nodes along the path between two nodes wishing to communicate. A route discovery table is maintained by the routers and the coordinator to implement route discovery. Zigbee uses the AODV and broadcast mechanism to reach the destination.

\subsubsection{Security in Zigbee}

The Zigbee protocol provides services at the NWK and APS layers to ensure the following security features: key establishment, secure networks, key transport and frame security. The Zigbee stack relies on an open trust model; hence, each layer trusts the others.

Zigbee network security is ensured by two encryption keys: the network key and the link key. The network key is used to secure broadcast communications. This 128bit key is shared amongst all devices in the network. There are two ways for a device 


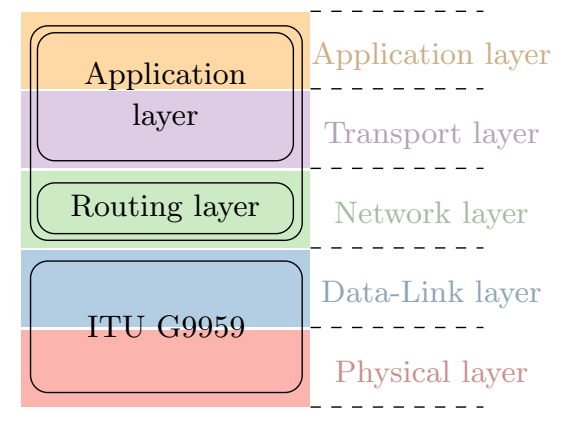

Figure 7: Z-Wave stack compared with the generic IoT stack. International Telecommunication Union (ITU)

to acquire this key, either by preinstallation or by key transport. The link key is used to secure unicast communication on APL. Hence, only two devices share a 128-bit key. A link key can be acquired by preinstallation, key transport or key establishment.

Because Zigbee is based on an open trust model, each layer provides its own frame security. The NWK layer provides a 128-bit key with AES-CCM to ensure encrypted communication and uses a cipher block chaining message authentication code (CBCMAC) to ensure integrity. APS guarantees the security of its frame using either the network key or the link key. If the former should be used, then APS checks whether the NWK layer already provides security; if it does so, then APS does not add security on its frame.

\subsection{Z-Wave}

LAN Close No Star, Mesh No

Z-Wave is a proprietary home-automation protocol developed by Zensys in 1999. It was later bought by Sigma Designs. Unlike most LAN protocols, Z-Wave operates on a subgigahertz band. This protocol is fully proprietary; however, some parts of the stack have been reverse engineered [63] to provide open-source solutions compatible with Z-Wave devices, such as OpenZwave. ${ }^{1}$

\subsubsection{Z-Wave stack description}

Figure 7 highlights the two-part division of the protocol, with the International Telecommunication Union (ITU) G9959 recommendation for the lowest layers and the remaining layers within a proprietary specification. We can split the Z-Wave protocol in two layers, with an NWK layer that consists of the routing layer and an application layer that includes the transport layer and the application layers [64].

PHY layer. The PHY layer defines three debt rates, depending on the modulation used: 9.6 and $40 \mathrm{Kbps}$ using frequency-shift keying (FSK) modulation and $100 \mathrm{Kbps}$ using GFSK. Therefore, the frequency bands differs depending on the region in which ZWave is used. In Europe, for instance, Z-Wave operates at $868.42 \mathrm{MHz}$.

\footnotetext{
${ }^{1}$ http://www.openzwave.com/home
} 
MAC layer. The MAC layer handles data exchange between devices and is responsible for such actions as frame acknowledgement, retransmission and packet origin authentication. The MAC frame is split into three parts; the MAC header (MHR), the MAC service data unit (MSDU) and the MAC footer (MFR). MHR contains important information, such as HomeID and SourceID, in which HomeID is a unique 4-byte number identifying a network and sourceID is an 8-bit number identifying a node within the Z-Wave network.

The MAC layer defines two types of devices in the network: the control device and the end device. Control nodes initiate commands and end devices respond to these commands. Moreover, a control device can act as a primary controller or a secondary controller. The primary controller is unique to each network and assigns HomeID and SourceID for each Z-Wave node during the enrolment process. It also stores and maintains the routing tables for the entire network. The secondary controller contains the same HomeID as the primary controller and maintains the routing table. End devices with batteries can also forward messages to nodes out of range of the control node.

Routing layer. The routing layer ensures the success of message transmission between the control node and the end devices. Moreover, this layer specifies the maximum number of nodes as 232 and the maximum number of hops as four.

The routing layer is in charge of the routing features of the Z-Wave network. It assigns the role of each device with a unique primary controller, which manages the entire network. It also determines which end devices participate in routing. Therefore, it is responsible for managing network topology and maintaining the routing table, with features such as topology discovering and topology healing.

Application layer. The application layer depends on the Z-Wave application developer. Nevertheless, some behaviours are applicable to all devices. The application layer is responsible for executing received commands. Commands are split into two classes: device and command.

The command class is related to a specific function, whereas the device class is related to the device. The device class is separated into three subclasses: basic, generic and special. The basic class identifies controllers and end devices with or without routing capabilities. The generic class specifies the functions a device can perform within its own role. Finally, the special class defines more specific features in device functionality.

\subsubsection{Routing in Z-Wave}

Z-Wave is a mesh networking protocol composed of a unique primary controller, which manages the entire network, and up to 231 end devices, many of which have routing capability. Moreover, Z-Wave uses a source-routed mesh network architecture to create the routing in the network, in which the routing table is handled by the primary node and maintained by all control nodes. Hence, each control node can initiate communication with other nodes using the route stored in the routing table. A route can pass a maximum of four nodes to reach the destination. If a route is unavailable, then the source node attempts to reach the destination via another route, continuing these attempts until the path to reach the destination node is found. Then, the routing table is updated by the control node to add or remove unused routes. 


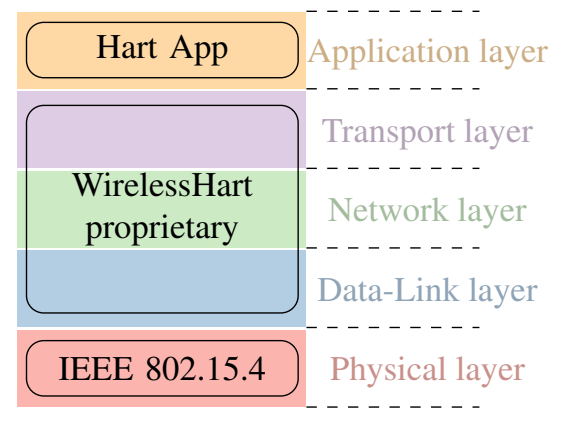

Figure 8: WirelessHart stack compared with the generic IoT stack.

\subsubsection{Security in Z-Wave}

Z-Wave comes without encryption by default, so all communications are sent in plain text which facilitates interception, gathering and decoding of messages. Until the fifth generation of Z-Wave devices, the only security features were HomeID, which uniquely identifies a network, and the explicit action required of the controller to add a new device to the network. In the latest generation (the 500 series), Sigma Designs introduced a secure pairing process and provided hardware capabilities supporting the 128-bit advanced encryption standard (AES-128). Once a device and the controller are paired using the secure mode, a key is exchanged and used to encrypt further communications. However, the latest generation must maintain backward compatibility with older devices that lack the same level of security. In addition, even if the newest devices can use a secure mode and encryption, older devices require a no-security level to operate. Therefore, legacy devices constitute an exploitable weak link.

\subsection{WirelessHart}

LAN Close No Mesh No

The WirelessHart [65] protocol has been designed solely for the process automation and manufacturing industries. It was created in September 2007 and relies on the already-popular highway addressable remote transducer (HART) protocol. This protocol was created to be suitable for IoT networks based on criteria such as power consumption and scalability just like all other IoT protocols. However, WirelessHart goes beyond these other protocols, adding reliability and security as key features of the protocol.

Figure 8 represents the stack of the WirelessHart protocol compared with our generic IoT stack. As explained earlier, the lower layer of WirelessHart is based on the open IEEE 802.15.4 standard. However, only the physical layer uses the IEEE standard; the data-link layer relies on other mechanisms specific to WirelessHart.

WirelessHart implements its own TDMA data-link layer and uses the concept of a superframe to provide better resistance to collision, as well as to provide deterministic communication.

According to [65], the network layer includes the transport layer and supports important features that ensure reliability against interference and obstacles. 


\begin{tabular}{|c|c|c|c|c|c|c|c|c|c|}
\hline & \multicolumn{2}{c}{ DLPDU footer } \\
\hline $0 \times 41$ & $\begin{array}{c}\text { Address } \\
\text { Specifier }\end{array}$ & $\begin{array}{c}\text { Sequence } \\
\text { Number }\end{array}$ & $\begin{array}{c}\text { Network } \\
\text { ID }\end{array}$ & $\begin{array}{c}\text { Dest. } \\
\text { Address }\end{array}$ & $\begin{array}{c}\text { Source } \\
\text { Address }\end{array}$ & $\begin{array}{c}\text { DLPDU } \\
\text { Specifier }\end{array}$ & $\begin{array}{c}\text { Data Link Layer } \\
\text { Payload }\end{array}$ & MIC & CRC \\
\hline
\end{tabular}

Figure 9: WirelessHart DLPDU structure.

The application layer is the same as that used in the HART protocol. Hence, it assures a perfect connection and compatibility between the two protocols, allowing a wireless device to communicate with another device outside of the WirelessHart network without modification of the packets.

\subsubsection{WirelessHart stack description}

As shown in Figure 8, WirelessHart defines its own data-link and network or transport layers to adapt the existing HART protocol to wireless devices.

Data-link layer. In WirelessHart, this layer fills the important role of providing reliable and secure communication. These mechanisms are established by the data link protocol data unit (DLPDU).

Moreover, as shown in Figure 8, the data-link layer differs from the data link provided by the IEEE 802.15.4 standard. It introduces two features, frequency hopping and channel blacklisting, to ensure reliability of communication.

Furthermore, TDMA used for the MAC layer improves resilience against collision by allowing transmissions only within a strict time slot of $10 \mathrm{~ms}$. Hence, multiple time slots are grouped to from a superframe. A superframe is used to control the timing transmissions.

The DLPDU of WirelessHart is detailed in Figure 9. The first byte is fixed to the $0 \times 41$ for every frame of WirelessHart. The TDMA data-link layer specification defines five types of frames:

- Acknowledgement DLPDU: This frame informs a sender of a nonbroadcast frame as to whether the frame was accepted.

- Advertise DLPDU: This frame contains all information needed to join the network. This frame corresponds to an invitation.

- Keep-alive DLPDU: This frame lacks a payload and is used for network time synchronisation or access communication between neighbours.

- Disconnect DLPDU: This frame advices all device neighbours that a network device is leaving.

- Data DLPDU: This is the only DLPDU that can contain information from a higher layer in the payload. All other DLPDUs are exclusively used at the second layer. 


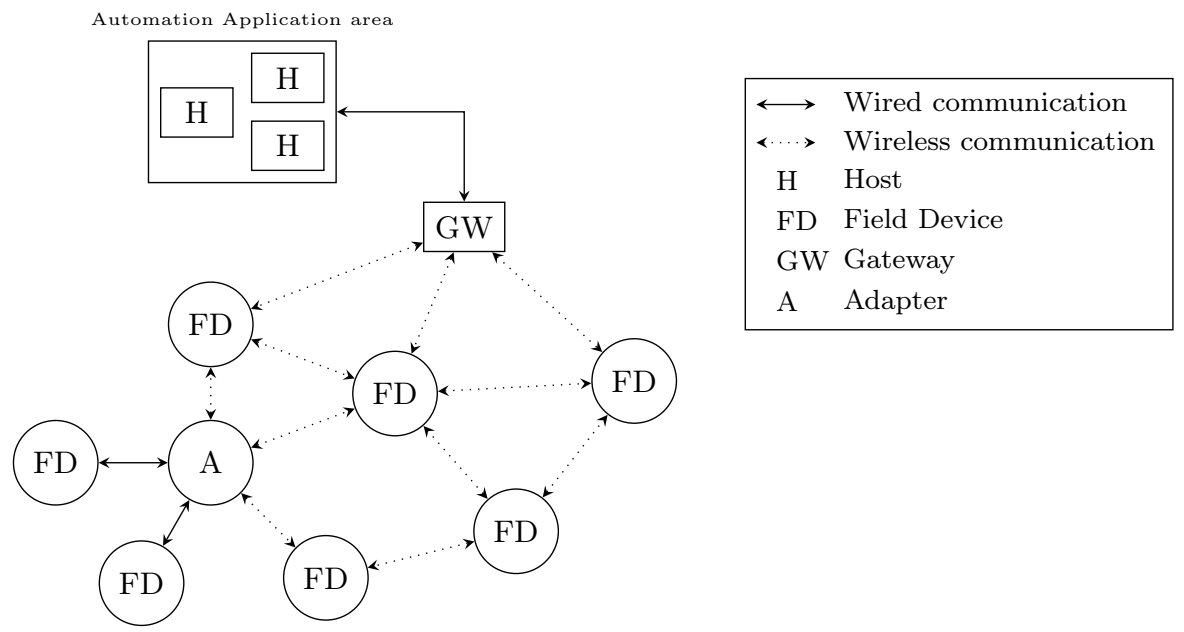

Figure 10: WirelessHart network structure.

Network and transport layers. The network layer is responsible of routing functionalities, as well as addressing and delivery of data in a secure manner. The transport layer controls reliable transmission and is included within the network layer [65]; it handles the security and reliability of the network.

The network manager is an important component of a WirelessHart network. It is responsible for forming and configuring the network, scheduling communication between devices, managing routing in the network and monitoring and reporting on the health of the network. There is only one network manager per WirelessHart network.

The network manager assures the functionalities provided by the network layer. All configurations of routing tables for the joining processes of network devices are handled by the network manager. Furthermore, it is the only component that can update the routing tables. There are two ways to provide routing according to WirelessHart standard [66].

\subsubsection{Routing in WirelessHart}

WirelessHart network provides many types of topology [65], including star, cluster and mesh. However, only the mesh topology is recommended for a WirelessHart network. In this network architecture, each device has the capability to provide routing, improving the scalability and reliability of the network. Moreover, a mesh topology provides redundant paths, assuring messages are routed around physical obstacles, broken links and various types of interference. All features are managed by a unique central point called the network manager. Figure 10 illustrates the WirelessHart network structure.

WirelessHart provides three mechanisms to handle messages routing: graph, source and superframe.

Graph routing. All paths to route a message from a source to a destination using the graph routing protocols are precomputed and form different graphs identified by a 
graph identifier (graph ID). These paths are created by the network manager and downloaded to each device. Thus, to send a packet, the source device includes in the header the specific graph ID determined by the destination. Hence, as each device proceeds to the destination, it knows the next node to which it should forward the packet.

Source routing. Unlike the graph routing protocol, the source routing protocol is based on an ad hoc protocol to create the route for messages. Thus, to send a packet, the source device provides a list of devices through which the packet must travel. When the packet reaches a node, it is forwarded to the next hop until it reaches the destination node. This protocol is used to provide network diagnostics.

Superframe routing. The superframe routing protocol is a variation of the graph routing protocol. The packets are assigned to a superframe. Whereas graph routing provides end-to-end routing paths in the network, superframe routing [67] uses the assigned superframes based on a graph to provide communication opportunities.

\subsubsection{Security in WirelessHart}

WirelessHart provides no choice as whether to use its security and disables the option of turning it off. Security services are handled at the MAC and NWK layers, and each layer ensures security features.

The MAC layer provides hop-to-hop security and data integrity using the message integrity code (MIC) with AES-128 in CCM mode. The network layer uses a set of keys to ensure security for end-to-end communication including confidentiality and data integrity. There are four keys:

- Public key: Used to generate MIC on the MAC layer by joining devices

- Network key: Shared amongst all devices and used to authenticate messages on a one-hop basis

- Join key: Unique to each network device and used to authenticate the joining device during the joining process with the network manager

- Session key: Generated by the network manager, unique for each pair of devices, and used to ensure confidentiality and data integrity for unicast communication.

\subsection{LoRaWAN}

WAN Close No Cellular No

LoRaWAN is a low power wide area network (LPWAN) intended for devices with constrained resources operating on a long-range network. LoRaWAN is a MAC layer protocol based on the long-range (LoRa) RF protocol (Figure 11) and maintained by the LoRa Alliance.

\subsubsection{LoRaWAN stack description}

LoRaWAN [68] is a protocol developed to be used with the LoRa modulation technique. It provides to features suitable for LPWAN. 


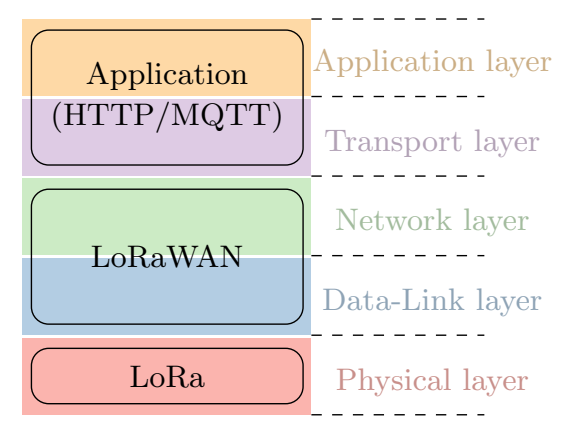

Figure 11: LoRaWAN stack compared with the generic IoT stack.

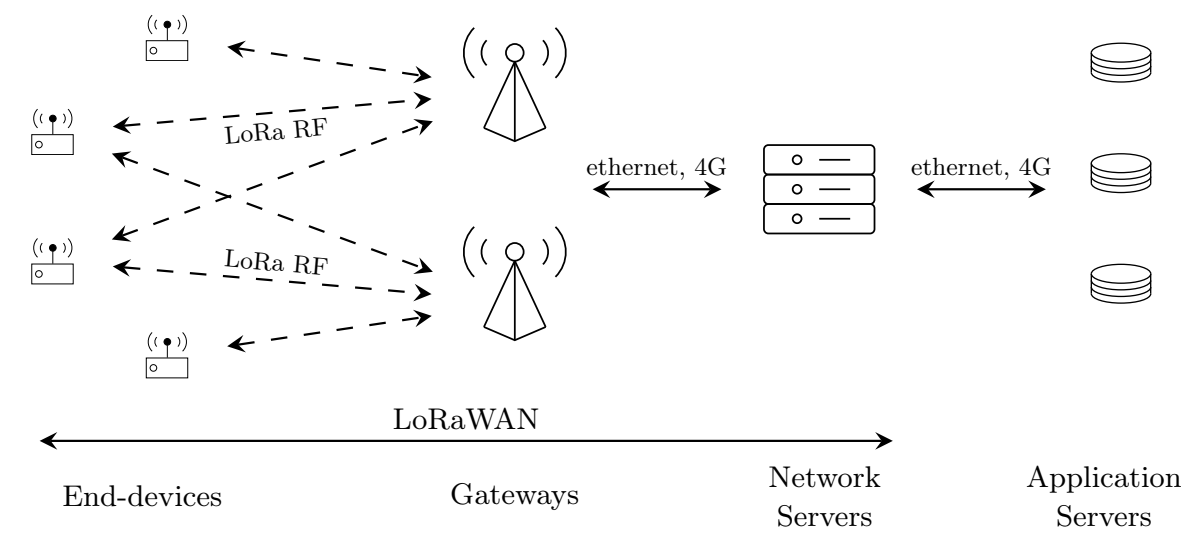

Figure 12: Architecture of a LoRaWAN network.

LoRa. LoRa is a proprietary radio modulation of the chip spread spectrum for LPWAN. It uses the subgigahertz RF bands $(868 \mathrm{MHz}$ in Europe and $915 \mathrm{MHz}$ in North America). It provides a long communication range, typically around $5 \mathrm{~km}$ in urban areas and up to $50 \mathrm{~km}$ in rural areas.

LoRaWAN. LoRaWAN defines the system architecture (shown in Figure 12) of the network and the communication protocol. Moreover, LoRaWAN enables bidirectional communication and thus defines three classes of devices:

- Class A: Class A must be supported by all devices. This is the class with the lowest energy consumption but with high downlink latency. Only two short windows for receiving downlinks are available after an uplink transmission of an end device.

- Class B: Class B devices include all class A windows for receiving downlinks and open additional windows for receiving downlinks at scheduled times. A gateway always sends a time-synchronised beacon to the end device. Thus, the server always knows when the device is listening. 
- Class C: Class C consumes the most energy of all classes. However, devices from class $\mathrm{C}$ have continuously open windows for receiving downlinks except when the devices are transmitting.

Each class serves a particular application need and has optimised requirements for a specific use. The difference amongst the three classes is the relationship between power consumption and latency. Class A has the lowest energy consumption but the highest latency, whereas class $\mathrm{C}$ offers the lowest latency but needs a high-energy power source.

\subsubsection{Routing in LoRaWAN}

The architecture of a LoRaWAN network comprises four parts, as shown in Figure 12. From left to right, end devices communicate only with the gateway using LoRa RF protocol. Then, gateways centralise all messages from end devices before transmitting them to the network servers using high-data-rate technologies like Ethernet or long-term evolution $(4 \mathrm{G})$. In a similar manner, the network servers transfer data to application servers using high-data-rate technologies. LoRaWAN uses a cellular topology, also known as star of stars (Figure 2).

Moreover, each end device is associated with multiple base stations (or gateways). Hence, all data sent by the devices are forwarded by multiple gateways, without regard for redundancy or the integrity of the data. These verifications are applied by the network server. The network server is in charge of the validation of various characteristics, such as data redundancy, integrity verification, receipt confirmation, emission power and even the debt adaptation.

\subsubsection{Security in LoRaWAN}

LoRaWAN defines three security keys that provide encryption and authentication in the network: the network session key (NwkSKey), the application session key (AppSKey) and the application key (AppKey). Each key has a 128-bit length. The NwkSKey is used to communicate at the network level (device to network servers). This key is also used to compute the MIC of each message sent on the network to ensure its integrity. The AppSKey is used to encrypt messages at the application level. This means that only the end device or the application server can encrypt and decrypt the messages. NwkSKey and AppSKey are unique to each device and to each session and are generated according to the device activation method, either dynamically or statically. The over-the-air-activation (OTAA) method uses the AppKey and obtains other resources from the server to dynamically derive the set of keys. The static method (ABP) uses a preshared AppKey to derive the two session keys. Based on the method used, NwkSKey and AppSKey are regenerated whenever the device is dynamically activated (OTAA); otherwise, the sessions keys remain the same until changed.

\subsection{Sigfox}

WAN Close No Cellular No

Sigfox is another LPWAN technology that was created before LoRaWAN in 2009. These two protocols are intended for long-range communication and resource-constrained devices but provide different solutions to achieve their goals. Sigfox acts as an operator 


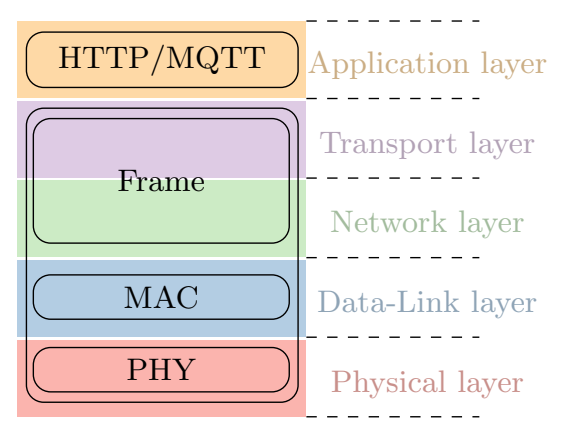

Figure 13: Sigfox stack compared with the generic IoT stack.

that owns its network; hence, a subscription to the Sigfox operator is required to use the network.

Sigfox is a proprietary protocol that includes all layers except the application layer (as shown in Figure 11).

\subsubsection{Sigfox stack description}

Sigfox uses ultranarrowband radio technology and as LoRaWAN operates on the ISM bands ( $868 \mathrm{MHz}$ in Europe). A specific feature of Sigfox is a payload length of only 12 bytes. The protocol is designed to be used with a short payload. Moreover, the use of the ISM band restrains the number of packets sent each day. Hence, the limit on packets per device in Sigfox is 14 messages per day. Thus, the technology is mainly used by applications that do not require a continuous flow of data.

According to Figure 13, the Sigfox stack includes three parts: the PHY layer, the MAC layer and the frame layer.

PHY layer. The role of the PHY layer is the same as for all other protocols: handle the protocol signal. In other words, the PHY layer manages RF modulation. Sigfox uses two modulation algorithms, depending on the type of communication: differential BPSK (DBPSK) modulation for uplink and GFSK modulation for downlink.

This layer is also in charge of the bit rate and the operation frequencies, depending on the country in which Sigfox is used. For example, in European countries, the Sigfox protocol operates on the $868-\mathrm{MHz}$ band at a bit rate of $100 \mathrm{bps}$.

MAC layer. The MAC layer is in charge of the device's authentication in the network. To do this, the MAC layer adds fields for authentication, as well as other standard programmes such as an error-detection code (cyclic redundancy check). Moreover, the Sigfox protocol removes all signalling from the MAC layer and devices. This means devices are not synchronised with the network.

Frame layer. The frame layer includes the transport and network layers from the generic IoT stack. The frame layer must satisfy the functionalities of these two layers. In other words, it acquires the payload provided by the application layer and generates the radio frame that is useful for the lower layers. Therefore, the frame layer adds a sequence number systematically into the frame. 


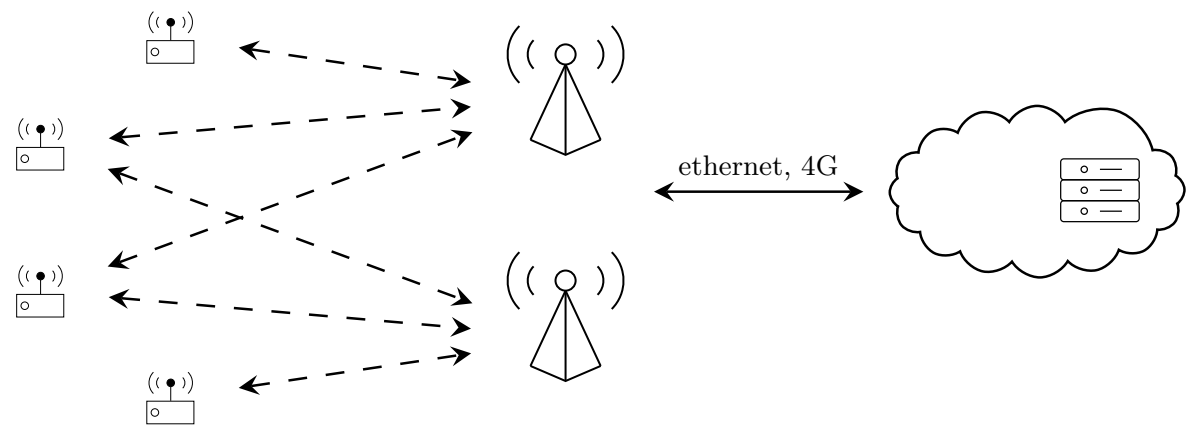

$\begin{array}{lr}\text { End-devices } & \text { SigFox } \\ \text { Gateways } & \text { Cloud }\end{array}$

Figure 14: Architecture of a Sigfox network.

\subsubsection{Routing in Sigfox}

The routing in Sigfox is similar to the routing in LoRaWAN. The topology of a Sigfox network is also a star of stars, as shown in Figure 14. However, Sigfox uses a cloud platform to transfer the processing power, rather than transferring in the devices, to reduce the energy consumption.

Devices enable bidirectional communication, but downlink communication can only be started by the device. Then, a short window is enabled for the downlink. Moreover, like LoRaWAN, Sigfox devices are not associated to a unique base station. The data can be sent through multiple antenna to reach the cloud platform on which the data will be used.

\subsubsection{Security in SigFox}

Sigfox devices are provisioned with a unique symmetrical AES-128 authentication key. This key is derived to provide a MAC for each message to ensure authenticity and integrity. In addition, a sequence counter avoids replay attacks. However, there is no confidentiality mechanism; by default, all messages are sent in plain text. Therefore, applications must implement their own end-to-end encryption to protect critical information. Sigfox clearly relies on security through obscurity, providing a subpar security quality, while denying access for research on the proprietary protocol [69].

\section{IoT attack study}

IoT devices are embedded devices that include operating system and system software. Moreover, these devices are interconnected and use wireless communication to exchange messages. These features all imply the likehood of IoT security threats, which can be evaluated using the traditional confidentiality, integrity and availability principle:

- Confidentiality: Capacity to hide information from those who are not authorised to view it. 
- Integrity: Capability to ensure that transmitted data are not tampered with or modified from the source to the destination

- Availability: Capacity of information to always be readily accessible by entities authorised to access it. For the rest of this paper, when we talk about attacks against availability, we only refer to attacks implemented by an attacker, not those caused by natural disasters.

We consider IoT systems to be networks of devices with a specific architecture, or topology. Every device can exchange data with every other device through communication using one or multiple protocols. Moreover, even though there can be many software issues in IoT devices, we focus on vulnerabilities against communication and protocols. Software issues are usually not specific to IoT but rather are classical IT problems.

Focus on packets

Focus on protocols

Focus on the system

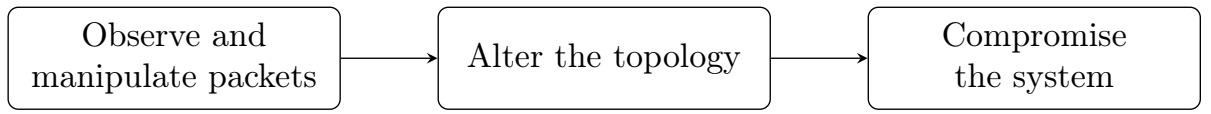

Figure 15: IoT kill chain.

We propose in Figure 15 an attack pattern that an attacker could follow to gain full control of an IoT system. This kill chain is split into three parts: packets, protocol and finally the system. The first step is focused on devices and more precisely, on sent and received messages (Section 5). The second step of the kill chain concerns the protocol and possible ways to alter the topology to control a subnet or the entire network (Section 6). The last step describes attacks against the whole system after it has been compromised (Section 7).

Table 3 summarises all attacks presented and detailed in this sections for all protocols. When an attack is possible in theory or practically for each protocol, the cell is filled with one or more references. Empty cells show attacks for which there is not enough documentation available, either on the protocols or on the attack, to be able to identify whether the attack is feasible. Finally, when we think that an attack cannot be achieved, we place a $\boldsymbol{X}_{\text {in }}$ the corresponding cell. The assumption of whether an attack is possible if no reference is available depends on analysis of the protocols and our experience. 


\begin{tabular}{|c|c|c|c|c|c|c|c|c|}
\hline \multicolumn{2}{|c|}{ Attacks Protocols } & OS4I & BLE & ZigBee & Z-Wave & WirelessHart & LoRaWAN & SigFox \\
\hline \multirow{2}{*}{$\begin{array}{l}\frac{n}{\tilde{u}} \\
\frac{\tilde{y}}{\tilde{D}} \\
\end{array}$} & Passive cryptographic & [70] & {$[18,71,72]$} & [73] & [17] & [19] & {$[74,75]$} & [69] \\
\hline & Active cryptographic & [76] & [77] & {$[73,78,79]$} & [17] & & {$[74,75]$} & [69] \\
\hline \multirow{6}{*}{$\begin{array}{l}\frac{0}{0} \\
0 \\
0 \\
0 \\
0\end{array}$} & MITM & & [77] & [20] & [80] & & & \\
\hline & Flooding & {$[76,81,82]$} & & [73] & & [19] & [75] & \\
\hline & Sybil & [82] & $x$ & [83] & & $x$ & $x$ & $x$ \\
\hline & Spoofing & [84] & & [73] & & [19] & {$[74,75]$} & \\
\hline & Wormhole & {$[85,82]$} & $x$ & {$[20]$} & & [19] & [86] & \\
\hline & denial-of-service (DoS) & [87] & & {$[88,79]$} & {$[80,17]$} & [19] & {$[74,75]$} & \\
\hline \multirow{2}{*}{ 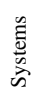 } & Sinkhole & [82] & $x$ & {$[89,90]$} & & & $x$ & $x$ \\
\hline & Selective forwarding & [82] & $x$ & [20] & & [19] & $x$ & $x$ \\
\hline
\end{tabular}

Table 3: Attacks summary.

\section{Focus on packet security}

Packet security is the first element in the kill chain as shown in Figure 15. This step consists of retrieving information or potentially gaining control of a device. As described earlier, we deliberately only consider vulnerabilities against communication and protocol and not software exploitation. Thus, this step describes attacks against communication from and to a device - in other words, cryptographic attacks. We distinguish passive cryptographic attacks from active ones.

Passive cryptographic attacks consist of extracting secrets or confidential data from intercepted frames without emitting a message. Because no action is performed within the network, there is no risk of altering the integrity or availability of the network. Instead, passive cryptographic attacks compromise confidentiality by stealing information from devices through eavesdropping.

With active cryptographic attacks, an attacker can modify messages transmitted through the network. The attacker can also inject traffic by forging or replaying frames to disturb the network. Hence, an active attack aims to compromise the three principles of confidentiality, integrity and availability.

\subsection{Passive cryptographic attacks}

Wireless communications are natively prone to traffic interception. Encryption is one solution to ensure that transmitted data are protected against unauthorised access. We distinguish four levels of cryptographic attacks, which we characterise as attacking no cryptography, ugly cryptography, bad cryptography and good cryptography.

\subsubsection{No cryptography}

Many IoT systems use unencrypted communications to communicate. This makes it easy to gain information from captured packets. In this case, no specific actions are required from the attacker to obtain the information contained in a message: the attacker examines the wireless communications and extracts information without computing actions. 


\subsubsection{Ugly cryptography}

We consider the cryptography to be ugly when communications are encrypted with weak cryptography, such as an old algorithm or small keys, and are thus easily breakable. ${ }^{2}$ In this case, an attacker can obtain all plain texts from encrypted communications using limited computational power. Communications with ugly cryptography should be considered equivalent to those that are unencrypted.

\subsubsection{Bad cryptography}

With bad cryptography, the algorithms that encrypt the payloads use state-of-theart standards, but the developers relied on an ad hoc implementation or deployment of these algorithms. Often, these implementations do not tackle every aspect necessary to provide the expected level of security. Weaknesses may lie in key generation, key exchange or key encryption, etc.

In [17], the authors, after analysis of the frame encryption and authentication algorithm in a Z-Wave network, were able to find a vulnerability that allowed them to take the control of a smart door. It appears that the encryption key was not sent in pain text. Hence, the first thing an attacker must determine is how the key is encrypted. The authors assumed that the key was generated by a pseudo random number generator (PRNG) provided by the Z-Wave chip and then encrypted with a hard-coded temporary default key in the chip's firmware. They found that this key value was 16 bytes of zero and they were able to retrieve the plain text of the key.

\subsubsection{Good cryptography}

In good cryptography, the algorithms used to encrypt communications are strong enough to be hard or impossible to break in a finite time. This cryptography relies on a standard algorithm, such as AES, with good parameters, sufficient key size, valid implementation and proven deployment. Here, an attacker may not to be able to find the plain text of the payload, yet it may still be possible to identify patterns that allow the attacker to gain some information.

\subsection{Active cryptographic attacks}

Good implementation of a cryptographic standard is not always enough to ensure perfect security of data. Other mechanisms are important to secure communication within a network. However, to test whether these mechanisms are present, an attacker must inject packets into the network or tamper with existing packets in the network.

\subsubsection{Same-nonce attacks}

In cryptography, nonces are random numbers that must be used only once. These numbers can be generated from a random or a pseudorandom generator. The main goal of these nonces is to avoid the reused of a frame in a replay attack. A same-nonce attack allows an attacker to retrieve partial information regarding plain texts from cipher text messages.

\footnotetext{
${ }^{2}$ https://www.wired.com/story/hackers-steal-tesla-model-s-seconds-key-fob/
} 
This attack is possible in Zigbee [91, 79, 78] and 6LoWPAN [76], mainly because of the security provided by IEEE 802.15.4 at the MAC layer [23]. The cipher suite used in IEEE 802.15.4 to encrypt payloads is AES-CTR. Hence, the payload is XORed with a keystream generated from a nonce and a preshared key. The nonce is derived from the device's extended address and the frame counter. When two payloads are encrypted with the same keystream, it is possible to decrypt them. Thus, let's consider two payloads $P$ and $P^{\prime}$, their encrypted versions $C$ and $C^{\prime}$ and the $K$ keystream. We have $C \oplus C^{\prime}=(P \oplus K) \oplus\left(P^{\prime} \oplus K\right)=P \oplus P^{\prime}$. This conclusion is possible if the counter does not change between two frames.

In [76], the authors identified that when a device could not receive the expected beacon in time, the device rebooted. This unexpected behaviour reset the counter to zero but kept the same extended address and preshared key. Thus, in waiting for the good frame, it was possible to obtain several frames with the same keystream.

\subsubsection{Replay attacks}

A replay attack consists of simply resending intercepted frames to the same device a moment later. The consequences of a replay attack are diverse, such as command replays and data retransmissions. Therefore, a replay attack does not allow an attacker to gain new information from the intercepted frame; instead this attack represents thus an integrity issue (not a confidentiality one).

In [76], the authors used the same vulnerability as for a same-nonce attack: counter reset. Just as in the same-nonce attack, when the device rebooted, its counters were reset. Thus, it was possible for an attacker to replay an intercepted frame with the right frame number.

In the same manner, in [78], the authors showed how they could switch on a light by replaying a frame. In Zigbee, when a counter reaches the maximum number, it is reset to zero but neither the nonce nor the key used to generate the keystream is changed. Thus, to replay a frame, the attacker just had to wait until the counter was reset.

\subsubsection{Malleability attacks}

A malleability attack is a combination of the two previous attacks: replay and samenonce. In other words, in a malleability attack, an attacker forges a new frame, encrypts it and sends it through the network. In a same-nonce attack, the attacker retrieves plain text and with the plain text and its cipher text, can retrieve the keystream using an XOR operation: $C=P \oplus K \rightarrow K=C \oplus P$, where $C$ is the cipher text, $P$ is the plain text and $K$ is the keystream. With the keystream, it is possible to forge a new encrypted frame. Instead of using a replay attack, an attacker can send the new forged frame with the same counter.

In [76], the authors took advantage of counter reset after device reboot to use samenonce attacks and gain large amounts of cipher text encrypted with the same keystream. In this way, they could retrieve many plain text frames. Using the XOR operation between plain text and cipher text, they retrieved several keystreams with different counters. Thus, they had the capability to forge new frames with the keystreams. Then, instead of replaying an intercepted frame to pull off a replay attack, the authors sent their forged frames with the corresponding counter through the network. 


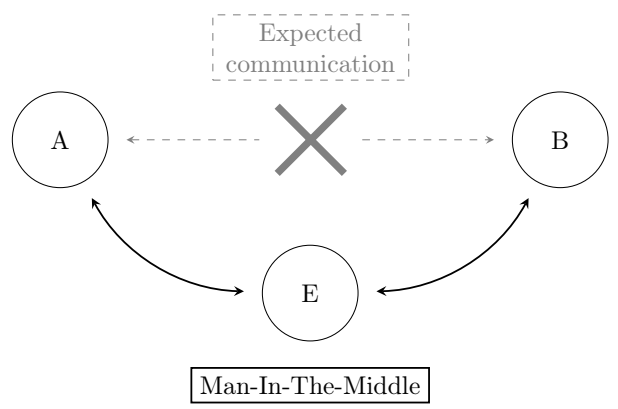

Figure 16: MITM attack.

\section{Focus on protocol security}

After the first step of the kill chain shown in Figure 15, an attacker was able to retrieve information or potentially take control of a device. The next step is to spread that control or simply increase the privileges. In an IoT system, this consists of altering the topology to take control of some part of the network using protocol weaknesses.

\subsection{MITM attack}

The MITM attack is well known in network and communication protocols. It consists of secretly intercepting, altering and relaying information between two devices that believe they are communicating with each other. Figure 16 represents the traditional scheme of an MITM attack, in which device A and device B are the two legitimate devices and device $\mathrm{E}$ is the attacker attempting to intercept the traffic.

In [92], the author presented a tool to perform an MITM attack against devices using the BLE protocol to communicate. During the presentation, he details the requirements needed to perform this attack and how easy it is to implement it. The attack consists of creating two fake devices, one acting as the central device and one as a dummy. The central device will connect to the legitimate device, and the dummy is a perfect copy in term of features and characteristics of the legitimate device. The dummy and the central device must be connected to each other to relay communication. The most important thing is timing. To perform this attack, it is mandatory that the attacker connect the central device to the legitimate device before the user can do so. Then, the attacker must copy the device to catch the user connection and relay information between the legitimate device and the user through the central device and the dummy. Therefore, the user believes communications with BLE-enabled device is occurring normally, but instead, all communications are being intercepted, analysed and even altered. Depending on the tool used to perform the MITM attack, different capabilities are available such as a replay attack or on-the-fly communication alterations.

\subsection{Flooding attack}

A flooding attack consists of sending a succession of requests to a particular device. This action attempts to consume enough of the device's resources to limit or stop 


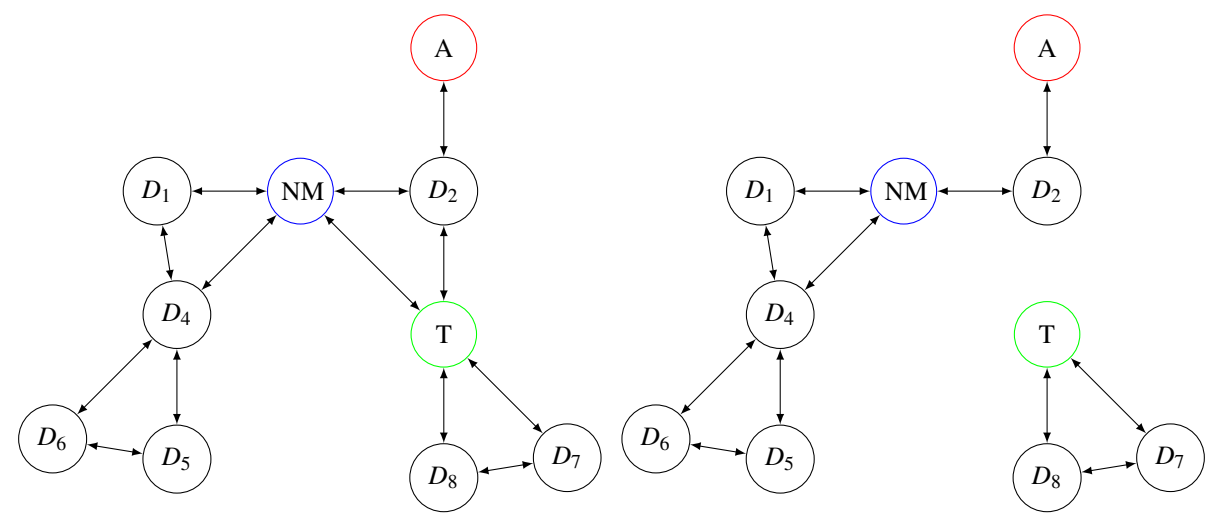

(a) Network before the disconnect attack

(b) Network after the disconnect attack

Figure 17: Network before (a) and after (b) the disconnect attack.

its activity. Furthermore, this attack can be divided into multiple attacks with different consequences, such as the Hello flood attack [82]. Generally, a flooding attack is considered the simplest attack leading to DoS [93, 94].

In [76], the authors flooded a 6LoWPAN sensor to make it lose synchronisation with the PAN coordinator. Device reboot was not an anticipated behaviour, but they found that sensors, because of the flooding attack, could not receive the expected beacon from the PAN coordinator, giving rise to loss of synchronisation. Thus, the sensor had to resynchronise with the PAN coordinator. By capturing the IEEE 802.15.4 frames sent to the coordinator, it was possible to gather useful information, such as the extended address of the device. Moreover, it appears that this flooding attack affected the PAN coordinator in a same manner, forcing it to reboot. Hence, this attack allowed not only the disruption of the sensor but also the gathering of information required to lead other attacks.

\subsection{Spoofing attack}

A spoofing attack is a well-known network security attack. It consists of an attacker successfully masquerading as another device by tampering with the target's data. In our case, a spoofing attack consists of using a controlled device to spoof a legitimate device inside a network to implement different actions, such as disconnecting or sending false information.

In [95], Bayou et al. implemented a spoofing attack to disconnect a node. This attack was based on the DLPDU structure, especially the disconnect type of DLPDU. This structure was created at the data-link layer and secured by the network key (shared by all network devices). According to the WirelessHart standard, this type of message allowed the device to inform its neighbours that it was leaving the network. Thus, each neighbour removed all information about the departing device from its neighbour list and deleted all links towards or from it. Then, the neighbours reported this device disconnection to the network manager, which updated the routing tables. 
To implement a disconnect attack, the attacker must spoof the identity of a legitimate node by forging a false disconnect DLPDU with the source address of the targeted device and then send it to the network manager. Afterward, the network manager will remove all links of the targeted device from its routing table. To perform this attack, the attacker needs a preparation state to gather information about the targeted device. The short address and the long address of the device are mandatory. Furthermore, the attacker's device must be synchronised with the network's current time because of the use of TDMA (see Section 3.5 for more details). This action can be done as follows:

- Compromise a legitimate device that received its own schedule from the network manager.

- Use the retry slot to send the disconnect packet to each target's neighbour one by one until the parent is found.

In [95], the authors used the join link to send the disconnect DLPDU. This link is normally used by new devices to join the network. But to accelerate the joining process, WirelessHart allows advertisement DLPDU to be sent on the join link. This message contains joining information and is sent periodically through the entire network. Hence, the attacker's device must wait for an advertisement DLPDU from the target's parent and use the join link to send the disconnect message.

Figure 17a represents the network before a disconnect attack, in which the red node is the attacker's device $A$, the green node represents the $\operatorname{target} T$ and the blue node is the network manager $N M$. During this phase, $A$ acts as a normal device. As soon as the attack is launched, $A$ enters search mode to gather information about the target and waits for an advertisement message. When the target parent $\left(D_{2}\right.$ in Figure 17) sends it, $A$ forges a disconnect DLPDU, sets the short address of $T$ as the source address and sends it to $D_{2}$. After receipt and validation of this packet with the network key, $D_{2}$ removes the sending device from its neighbour list and forwards the information to $N M$. Thus, all links with $T$ are removed and this device is no longer considered a device of the network (as shown in Figure 17b). All packets from $T$, whether its own or those of its children, are dropped. At this point, two distinct networks exist with no communication between them.

\subsection{Sybil attack}

The sybil attack [96] aims to forge and use numerous identities from a single malicious node. It has been widely used to target distributed reputation systems but is also a threat for distributed routing protocols. With numerous created identities, an attacker holds a large amount of influence in the network, which might affect the network's effectiveness and even the chosen paths of distributed service or multipath routing.

This attack is only possible if the network does not run a trusted central authority that verifies each identity before including it in the network. This lack of control is a traditional weakness of wireless ad hoc and sensor networks.

In [97], Medjek et al. described how an attacker could throw a sybil attack against RPL-based network. They used and took advantage of an RPL feature to add a node to the network. Two methods exist to join an RPL network: waiting for DODAG information object (DIO) messages or sending DODAG information solicitation (DIS) 
messages. These DIS messages are similar to router solicitation messages, allowing a node to discover DODAG information from its neighbours by soliciting DIO messages. Once an attacker receives DIO messages, the attacker fills a DODAG destination advertisement object (DAO) message with a crafted node identifier and its parent address. The attacker then broadcasts the DAO message to be advertised by the node neighbour to update the routing table and the parent list. With this method, the attacker can create many nodes with forged identities and add them to an RPL network.

\subsection{Wormhole attack}

In wormhole attacks [98], the attacker uses a tunnel between two malicious nodes to relay messages from zone A to zone B faster or with fewer apparent hops than would be done using the expected multihop protocol. The attacker can use a wired link between the two relays or even fast wireless connectivity. This manipulation allows the attacker to be seen as the best route between these two zones and thus to relay most packets between them. The attacker then gains privileges that allow eavesdropping or manipulation of the routed packets. This attack is highly dangerous, because it does not require known cryptographic keys, thus, this attack is effective even if the communication network provides confidentiality and integrity.

In [85], Perazzo et al. presented an implementation of a wormhole attack against a 6LoWPAN network built upon IEEE 802.15.4 and using the routing algorithm RPL. The first step of the attack was to enable communication between devices too distant to communicate directly. This was possible using two malicious nodes, $m n_{1}$ and $m n_{2}$, as gateways, one in each area and connected with a high-speed connection, such as the Internet or a local network. Another important point is the method of delivery for ACK packets to ensure that devices used the tunnel as shortest path to reach the destination. The time between emission and receipt of an ACK packet must be shorter than the timeout. Therefore, using a specification of IEEE 802.15.4, in which ACK packets must not be authenticated, the authors used a programme to reply to an ACK response for any unicast frames received in both malicious nodes. The attack was thus led as follows: $m n_{1}$ intercepted DIO broadcast messages from zone A and relayed them to $m n_{2}$, which replayed the messages in the other zone. Each device receiving the DIO message responded to the sender with a unicast frame. These frames were intercepted by $m n_{2}$, and then it automatically replied with a forged ACK packet before transferring the unicast frame to $m n_{1}$. In this way, the attacker was able to reduce the number of hops between the two devices and to control the traffic flow passing through the created tunnel.

\section{Focus on system security}

After the second step of the kill chain shown in Figure 15, an attacker was able to alter some parts of the topology. The attacker can then try to alter the functioning of the entire system. 


\subsection{Sinkhole attacks}

A sinkhole attack consists of creating a centralised point from a malicious node and luring all traffic from a particular area. Furthermore, all devices from this area must communicate through this malicious device. This attack makes it possible to perform many other attacks, such as selective forwarding.

To make the malicious device the centralised point for all other nodes, it must be seen as a good choice by respecting the routing algorithm. Depending on the algorithm used, the criteria to be an attractive node may differ. But a high-quality route and low-latency transmission are two points that allow it to become the central point of all communication. A perfect way to reach this aim is to spoof or replay an advertisement for a high-quality route to another device.

This attack is easy to implement in an RPL network [82] because of its particular architecture in the DODAG form. The attacker can intercept a DIO control message and change the advertised rank contained inside. The rank field identifies the position of the router inside the graph. Moreover, the attacker can remove the delay usually used to reduce network congestion. These two modifications increase the reputation of the malicious node, and force the other devices to send their traffic through this node.

\subsection{Selective-forwarding attacks}

A selective-forwarding attack takes place in networks with the assumption that any actives nodes are faithful when they forward packets. The selective-forwarding attack consists of allowing only wanted traffic to move through malicious nodes. The remaining packets are dropped, ensuring that they are not propagated.

There are two methods of implementing this attack. The first method is to use malicious nodes, becoming a black hole and forcing them to refuse to forward every packet. However, this behaviour may lead legitimate nodes to seek another route to send their packets. Hence, the second, more effective method consists of not dropping every packet but choosing only those the attacker wants to forward.

This attack, when applied against RPL [82], consists of letting the malicious nodes drop all packets except packets relative to the protocol. Hence, the attack insists that only packets destined for the malicious node or containing RPL information are not dropped. In this way, the protocol works normally, but application data can be lost. The RPL self-healing and self-management mechanisms cannot correct the malicious behaviour even after running for 24 hours [82]. 


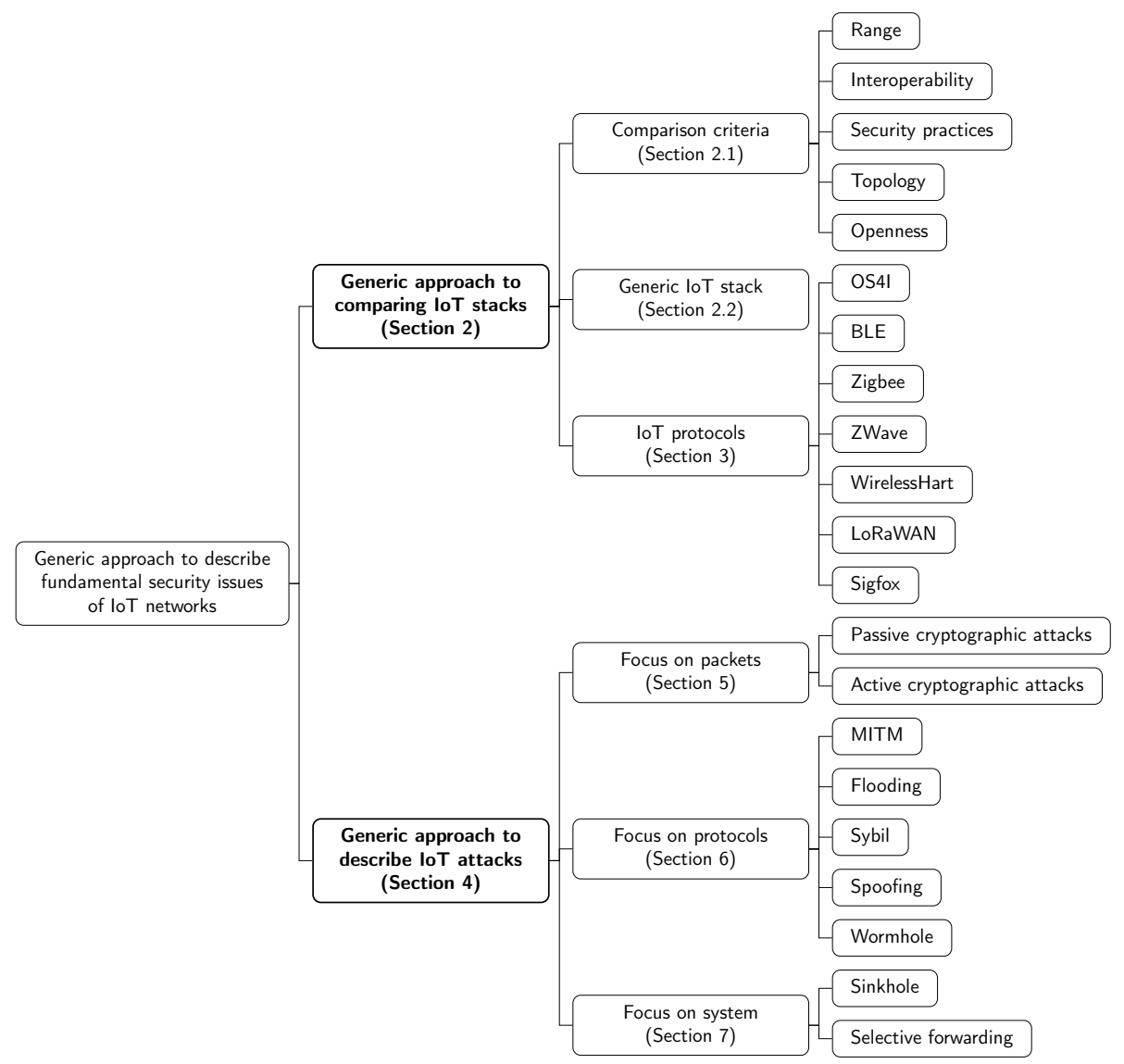

Figure 18: Summary of the generic approach to describe fundamental security issues of IoT networks.

\section{Discussion}

In this section, we discuss the lessons we learned from our approach to describe fundamental security issues of IoT networks and identify several initiatives for future work, which may improve the security of IoT networks.

\subsection{Approach to describe fundamental security issues of IoT networks: lessons learned}

Figure 18 outlines the approach we used to describe the security issues of IoT protocols. From this approach, we pinpointed two challenges we tackled in this survey.

The first challenge concerned the generic approach to compare different IoT stacks. From this challenge, we highlighted the difficulty of identifying common criteria and defining an abstract structure to efficiently compare different IoT protocol stacks. The significant related work presented in Section 1.3 emphasises the difficulty to characterize IoT protocol stacks generically. Depending on the area of activity, usages and applications, the characteristics and the specificities of IoT protocol stacks differ [99]. 
For instance, the Sigfox protocol, mainly used for wide-area communications with low data-rate, uses a DBPSK modulation for uplink and operates on the $868 \mathrm{MHz}$ band, whereas the BLE protocol, used for short-range communications with high data-rate, uses a GFSK modulation and operates on the $2.4 \mathrm{GHz}$ band. The availability of the documentation and specifications of every protocol is variable and accentuates the difficulty to provide a common approach to compare them. We tackled this challenge with the proposition of a generic IoT stack and five comparison criteria, which are used to present and analyse different IoT protocol stacks in a similar formalism. This proposition captures the fundamental characteristics of the IoT paradigm and, as such, should be able to capture future IoT protocols: we would need to classify these novel protocols according to the proposed criteria and, as our generic stack divides the protocols into fundamental IoT applications layers, we should be able to map future (versions of) protocols onto this stack.

The second challenge addressed the generic approach to describing IoT attacks. As described throughout this paper, published attacks against IoT protocols are always protocol-specific. We can explain this result with multiple points of view. The multitude of protocols makes the analysis of each attack against every protocols hard and tedious. Furthermore, regarding the protocol, the condition to realize the attacks could be hard or even impossible to reproduce in practice. For instance, two devices are required to perform a MITM attack in BLE, whereas an infrastructure must be deployed to ensure that Zigbee devices can communicate to finally perform the MITM attack. This constraint makes the generic analysis of attacks complicated. Finally, the availability and the accessibility of details about the security mechanisms differ with every IoT protocol stack, making the generic analysis of attacks hard for every IoT protocols stacks. We tackled this challenge with a killchain divided into three parts: attacks focusing on packets, attacks focusing on protocols and attacks focusing on systems. We were able to analyse each attack in relation to the formalism proposed for the generic IoT stack. Hence, we were able to inventory similar attacks on different protocols, link them, estimate which attacks could be similarly done against another protocol and which protocols were inherently resistant to specific attacks. In the event of a novel attack against a given protocol, we should be able to classify it in the killchain (packets, protocols, systems), identify whether it is an adaptation of an attack against another protocol or a fundamentally innovative one and, if it is an innovative one, analyse whether it could be used against other IoT protocols. Moreover, when new IoT protocols will arrive, once they are analysed through the lens proposed in the first part (comparison criteria, generic stack), we should be able to rapidly evaluate which vulnerabilities exist or, to the contrary, are mitigated.

\subsection{Perspectives to improve the security of IoT networks}

We can exhibit some root causes to these security problems. First, although protocol stacks evolve, commercial imperatives imply that they must keep some compatibility with already-deployed objects, which causes legacy security limitations $[100$, 101]. This problem may be mitigated by isolating parts of the system or by promoting open-source software or hardware, which usually carries fewer legacy requirements [102, 103], allowing old objects to be upgraded. Second, objects often cannot be 
upgraded [104], and for those that can vendors usually provide inadequate security policy upgrades [105] (which either are provided too rarely or are not provided for enough time, considering the expected duration of these objects). We strongly believe that IoT vendors need to foster communication with security researchers, rather than trying to mute them. These collaborations can lead to open specifications and collaborative bug bounties [106]. Third, commercial pressure puts usability before security [107], and any actor who would like to change this process would probably face lower sales than those of its competitors. A penetration testing approach [108] may yield exciting results by emphasising, for a given deployment, security measures that should not be ignored. Finally, despite providing similar functionalities, there are too many heterogeneous protocols. Security researchers and operator endeavours are thus scattered amongst systems, instead of uniting their forces. We need a common framework to mutualise security efforts and facilitate exchange amongst communities working on different protocols.

Starting from this work, we identify that a new method might be defined to ensure the same security mechanisms regardless of the protocol stack used. Defining this new method raises several new interesting research and methodological points:

- Identifying common security criteria by drawing up a list of security features amongst all protocols to establish a global base that could be applied for each protocol stack. Existing works $[109,110,111]$ provide classifications and taxonomies of security features that could be applied generically. However, these works are focused on a specific aspect or a specific application of the IoT and not on IoT protocol stacks. These works may be worth to be pursued to provide a taxonomy of security criteria suitable for every IoT protocol stacks.

- Harmonising security mechanisms by finding a solution to apply them regardless of the protocol stack used. A solution may be to define a certification that each protocol must apply $[112,113]$. This certification, relying on common security criteria, should be generic enough to suit the maximum IoT protocol stacks and cover the largest field of applications. We could imagine another solution, such as a framework [114] that identifies which security mechanisms must be applied according to the application domain targeted.

- Ensuring a constant security level, even if protocol stacks and deployments evolve. IoT environments are indeed much more subject to evolutions in time and space $[115,116]$. It means - in time - guaranteeing a regular follow-up of every version deployed for a protocol to ensure the same level of security. Systematic security regression testings need to be defined and need to also evolve with new IoT protocols. They should, for instance, avoid compatibility issues opening security breaches from two different versions of the same protocol. Or they should, for instance, avoid two different protocols known to be individually safe, but opening a security breach when combined. It also means - in space to be able to correlate a physical area and a security level. Deploying a new IoT device, a crash or misbehaviour of a device, should affect the security level and be considered as a potential intrusion. Automatic analysis of the device traffic should be able to dynamically qualify the level of security of the overall deployed 
IoT architecture. IoT environments require intrinsic flexibility to easily deploy anything, anywhere, anytime, but raise this new challenge to define new flexible security frontiers and guarantees in space and time.

Tomorrow's IoT will be multiprotocol, mixing various complementary systems deployed at different moments, and will have to deal with both the legacy of a common protocol and a range of different protocols. Analysis on the scale of a unique protocol will no longer be pertinent.

\section{Conclusion}

This survey aims to detail the many challenges to the security of IoT. We define a generic approach to compare major IoT protocol stacks, using a generic stack based on the OSI model. We base our comparison on five criteria: range, openness, interoperability, network architecture and security practices. We also suggest a kill chain against IoT networks, with multiple attacks that are not specific to a given protocol. Finally, we propose a comprehensive, structured and generic study of IoT security issues.

\section{References}

[1] A. Al-Fuqaha, M. Guizani, M. Mohammadi, M. Aledhari, M. Ayyash, Internet of things: A survey on enabling technologies, protocols, and applications, IEEE Communications Surveys \& Tutorials 17 (4) (2015) 2347-2376.

[2] L. Da Xu, W. He, S. Li, Internet of things in industries: A survey, IEEE Transactions on industrial informatics 10 (4) (2014) 2233-2243.

[3] J. Gubbi, R. Buyya, S. Marusic, M. Palaniswami, Internet of things (iot): A vision, architectural elements, and future directions, Future Generation Comp. Syst. 29 (7) (2013) 1645-1660.

[4] S. Sicari, A. Rizzardi, L. A. Grieco, A. Coen-Porisini, Security, privacy and trust in internet of things: The road ahead, Computer networks 76 (2015) 146-164.

[5] Gartner, Gartner says 5.8 billion enterprise and automotive iot endpoints will be in use in 2020, https://www.gartner.com/en/newsroom/pressreleases/2019-08-29-gartner-says-5-8-billion-enterpriseand-automotive-io, accessed on 17 June 2020 (2019).

[6] J. Lin, W. Yu, N. Zhang, X. Yang, H. Zhang, W. Zhao, A survey on internet of things: Architecture, enabling technologies, security and privacy, and applications, IEEE Internet of Things Journal 4 (5) (2017) 1125-1142.

[7] M. J. Kaur, P. Maheshwari, Building smart cities applications using iot and cloud-based architectures, in: International Conference on Industrial Informatics and Computer Systems (CIICS), IEEE, 2016, pp. 1-5. 
[8] T. H. news, How drones can find and hack internet-of-things devices from the sky, https://thehackernews.com/2015/08/hacking-internet-ofthings-drone.html, accessed on 21 August 2019 (2019).

[9] T. H. news, Z-wave downgrade attack left over 100 million iot devices open to hackers, https://thehackernews.com/2018/05/z-wavewireless-hacking.html, accessed on 21 August 2019 (2019).

[10] T. H. news, Blueborne: Critical bluetooth attack puts billions of devices at risk of hacking, https : //thehackernews.com/2017/09/blueborne-bluetoothhacking.html, accessed on 21 August 2019 (2019).

[11] T. H. news, Hackers could turn LG smart appliances into remote-controlled spy robot, https://thehackernews.com/2017/10/smart-iot-devicehacking.html, accessed on 21 August 2019 (2019).

[12] R. Hallman, J. Bryan, G. Palavicini, J. DiVita, J. Romero-Mariona, IoDDoS the internet of distributed denial of sevice attacks - A case study of the mirai malware and iot-based botnets, in: IoTBDS, 2017.

[13] J. Radcliffe, Hacking medical devices for fun and insulin: Breaking the human SCADA system, in: Black Hat, 2011.

[14] T. H. news, Hackers can remotely access syringe infusion pumps to deliver fatal overdoses, https://thehackernews.com/2017/09/hackinginfusion-pumps.html, accessed on 21 August 2019 (2019).

[15] T. H. news, Casino Gets Hacked Through Its Internet-Connected Fish Tank Thermometer, https://thehackernews.com/2018/04/iot-hackingthermometer.html, [Online; accessed on 21 August 2019] (2019).

[16] T. H. news, How to Hack Smart Bluetooth Locks and IoT Devices - Check this Out, https://thehackernews.com/2016/09/hacking-bluetoothlocks.html, [Online; accessed on 21 August 2019] (2019).

[17] B. Fouladi, S. Ghanoun, Security evaluation of the z-wave wireless protocol, Black hat USA 24 (2013).

[18] M. Ryan, Bluetooth: With low energy comes low security, in: 7th USENIX Workshop on Offensive Technologies, WOOT, USENIX Association, 2013.

[19] S. Raza, A. Slabbert, T. Voigt, K. Landernäs, Security considerations for the WirelessHART protocol, in: International Conference on Emerging Technologies and Factory Automation (ETFA), IEEE, 2009, pp. 1-8.

[20] P. Baronti, P. Pillai, V. W. C. Chook, S. Chessa, A. Gotta, Y. Hu, Wireless sensor networks: A survey on the state of the art and the 802.15.4 and zigbee standards, Computer Communications 30 (7) (2007) 1655-1695. 
[21] M. Sain, Y. J. Kang, H. J. Lee, Survey on security in internet of things: state of the art and challenges, in: International Conference on Advanced Communication Technology (ICACT), IEEE, 2017, pp. 699-704.

[22] K. T. Nguyen, M. Laurent, N. Oualha, Survey on secure communication protocols for the internet of things, Ad Hoc Networks 32 (2015) 17-31.

[23] A. Reziouk, E. Laurent, J.-C. Demay, Practical security overview of IEEE 802.15.4, in: International Conference on Engineering \& MIS (ICEMIS), IEEE, 2016, pp. 1-9.

[24] M. Brachmann, O. Garcia-Morchon, M. Kirsche, Security for practical CoAP applications: Issues and solution approaches, 10th GI/ITG KuVS Fachgespraech Sensornetze (FGSN11) (2011) 15-16.

[25] V. Karagiannis, P. Chatzimisios, F. Vazquez-Gallego, J. Alonso-Zarate, A survey on application layer protocols for the internet of things, Transaction on IoT and Cloud Computing 3 (1) (2015) 11-17.

[26] K. Lounis, M. Zulkernine, Attacks and defenses in short-range wireless technologies for iot, IEEE Access 8 (2020) 88892-88932.

[27] D. M. Mendez, I. Papapanagiotou, B. Yang, Internet of things: Survey on security and privacy, CoRR abs/1707.01879 (2017). arXiv: 1707.01879.

URL http://arxiv.org/abs/1707.01879

[28] A. Oracevic, S. Dilek, S. Özdemir, Security in internet of things: A survey, in: 2017 International Symposium on Networks, Computers and Communications (ISNCC), 2017, pp. 1-6.

[29] F. A. Alaba, M. Othman, I. A. T. Hashem, F. Alotaibi, Internet of things security: A survey, J. Network and Computer Applications 88 (2017) 10-28.

[30] M. Ammar, G. Russello, B. Crispo, Internet of things: A survey on the security of iot frameworks, J. Inf. Sec. Appl. 38 (2018) 8-27.

[31] K. Zhao, L. Ge, A survey on the internet of things security, in: International Conference on Computational Intelligence and Security (CIS), IEEE, 2013, pp. 663-667.

[32] G. Bora, S. Bora, S. Singh, S. M. Arsalan, Osi reference model: An overview, International Journal of Computer Trends and Technology (IJCTT) 7 (4) (2014) 214-218.

[33] B. A. Forouzan, TCP/IP protocol suite, McGraw-Hill, Inc., 2002.

[34] R. Ratasuk, B. Vejlgaard, N. Mangalvedhe, A. Ghosh, Nb-iot system for $\mathrm{m} 2 \mathrm{~m}$ communication, in: wireless communications and networking conference (WCNC), IEEE, 2016, pp. 1-5. 
[35] IEEE Standards Association, 802.15.4-2015 - IEEE Standard for Low-Rate Wireless Networks, Tech. rep.

URL https://ieeexplore.ieee.org/document/7460875

[36] J. Hui, D. Culler, S. Chakrabarti, 6LoWPAN: Incorporating IEEE 802.15. 4 into the IP architecture, Internet Protocol for Smart Objects Alliance (IPSO) (2009).

[37] J. Olsson, 6lowpan demystified, Tech. rep., Texas Instruments (2014). URL https ://www.ti.com/lit/wp/swry013/swry013.pdf

[38] J. Hui, P. Thubert, Compression format for ipv6 datagrams over ieee 802.15.4based networks, RFC 6282, RFC Editor (September 2011).

URL http: //www.rfc-editor.org/rfc/rfc6282.txt

[39] G. Montenegro, N. Kushalnagar, J. Hui, D. Culler, Transmission of ipv6 packets over ieee 802.15.4 networks, RFC 4944, RFC Editor (September 2007).

URL http: //www.rfc-editor.org/rfc/rfc4944.txt

[40] A. Ayadi, P. Maillé, D. Ros, L. Toutain, T. Zheng, Implementation and evaluation of a TCP header compression for 6lowpan, in: International Wireless Communications and Mobile Computing Conference (IWCMC), 2011, pp. 13591364.

[41] Z. Shelby, K. Hartke, C. Bormann, The constrained application protocol (coap), RFC 7252, RFC Editor (June 2014).

URL http: //www.rfc-editor.org/rfc/rfc7252.txt

[42] R. A. Rahman, B. Shah, Security analysis of IoT protocols: A focus in CoAP, in: International Conference on Big Data and Smart City (ICBDSC), IEEE, 2016, pp. $1-7$.

[43] C. Bormann, A. P. Castellani, Z. Shelby, CoAP: An application protocol for billions of tiny internet nodes, IEEE Internet Computing 16 (2) (2012) 62-67.

[44] N. Modadugu, E. Rescorla, The design and implementation of datagram TLS, in: Network and Distributed System Security Symposium (NDSS), 2004.

[45] T. Kothmayr, C. Schmitt, W. Hu, M. Brünig, G. Carle, DTLS based security and two-way authentication for the internet of things, Ad Hoc Networks 11 (8) (2013) 2710-2723.

[46] S. Raza, D. Trabalza, T. Voigt, 6lowpan compressed DTLS for CoAP, in: International Conference on Distributed Computing in Sensor Systems (DCOSS), IEEE, 2012, pp. 287-289.

[47] Andrew Banks and Rahul Gupta, MQTT version 3.1.1, OASIS Standard (29 October 2014).

URL http://docs.oasis-open.org/mqtt/mqtt/v3.1.1/mqtt-

v3.1.1.html 
[48] A. Ludovici, A. Calveras, J. Casademont, Forwarding techniques for IP fragmented packets in a real 6lowpan network, Sensors 11 (1) (2011) 992-1008.

[49] A. H. Chowdhury, M. Ikram, H.-S. Cha, H. Redwan, S. Shams, K.-H. Kim, S.W. Yoo, Route-over vs mesh-under routing in 6lowpan, in: International conference on wireless communications and mobile computing: Connecting the world wirelessly (IWCMC), ACM, 2009, pp. 1208-1212.

[50] C. Perkins, E. Belding-Royer, S. Das, Ad hoc on-demand distance vector (AODV) routing, RFC 3561, RFC Editor, http://www.rfc-editor.org/rfc/ rfc3561.txt (July 2003).

URL http://www.rfc-editor.org/rfc/rfc3561.txt

[51] S. D. Park, 6LoWPAN ad hoc on-demand distance vector routing (LOAD), Internet-Draft draft-daniel-6lowpan-load-adhoc-routing-03, IETF Secretariat (June 2007).

URL http://www.ietf.org/internet-drafts/draft-daniel-6lowpanload-adhoc-routing-03.txt

[52] T. H. Clausen, J. Yi, A. C. de Verdiere, Loadng: Towards AODV version 2, in: Vehicular Technology Conference (VTC), IEEE, 2012, pp. 1-5.

[53] O. Iova, G. P. Picco, T. Istomin, C. Király, RPL: the routing standard for the internet of things... or is it?, IEEE Communications Magazine 54 (12-Supp) (2016) 16-22.

[54] T. Winter, P. Thubert, A. Brandt, J. Hui, R. Kelsey, P. Levis, K. Pister, R. Struik, J. Vasseur, R. Alexander, Rpl: Ipv6 routing protocol for low-power and lossy networks, RFC 6550, RFC Editor (March 2012).

URL http://www.rfc-editor.org/rfc/rfc6550.txt

[55] J. Vasseur, N. Agarwal, J. Hui, Z. Shelby, P. Bertrand, C. Chauvenet, RPL: The IP routing protocol designed for low power and lossy networks, Internet Protocol for Smart Objects Alliance (IPSO) (2011).

[56] S. Raza, S. Duquennoy, T. Voigt, U. Roedig, Demo abstract: Securing communication in 6lowpan with compressed ipsec, in: International Conference on Distributed Computing in Sensor Systems and Workshops (DCOSS), IEEE, 2011, pp. 1-2.

[57] S. Raza, T. Voigt, V. Jutvik, Lightweight ikev2: a key management solution for both the compressed ipsec and the ieee 802.15. 4 security, in: Proceedings of the IETF workshop on smart object security, Vol. 23, Citeseer, 2012.

[58] B. SIG, Bluetooth specification version 4.0 vol 0 (6/2010), https: //www.bluetooth.org/docman/handlers/downloaddoc.ashx?doc_id= 229737, [Online; accessed on 21 August 2019]. 
[59] C. Gomez, J. Oller, J. Paradells, Overview and evaluation of bluetooth low energy: An emerging low-power wireless technology, Sensors 12 (9) (2012) 11734-11753.

[60] B. SIG, Bluetooth specification version 5.0, https://www.bluetooth.org/ DocMan/handlers/DownloadDoc.ashx?doc_id=421043, [Online; accessed on 21 August 2019].

[61] B. S. . M. W. Group, Bluetooth Mesh Networking specification, https: //www.bluetooth.com/specifications/mesh-specifications, [Online; accessed on 21 August 2019].

[62] S. M. Darroudi, C. Gomez, Bluetooth low energy mesh networks: A survey, Sensors 17 (7) (2017) 1467.

[63] C. W. Badenhop, S. R. Graham, B. W. P. Ramsey, B. E. Mullins, L. O. Mailloux, The Z-Wave routing protocol and its security implications, Computers \& Security 68 (2017) 112-129.

[64] C. W. Badenhop, J. D. Fuller, J. Hall, B. W. P. Ramsey, M. Rice, Evaluating ITU-T G.9959 based wireless systems used in critical infrastructure assets, in: 9th International Conference Critical Infrastructure Protection (ICCIP), 2015, pp. 209-227.

[65] D. Chen, M. Nixon, A. Mok, WirelessHART: Real-Time Mesh Network for Industrial Automation, 1st Edition, Springer, 2010.

[66] J. Song, S. Han, A. Mok, D. Chen, M. Lucas, M. Nixon, W. Pratt, Wirelesshart: Applying wireless technology in real-time industrial process control, in: RealTime and Embedded Technology and Applications Symposium (RTAS), IEEE, 2008, pp. 377-386.

[67] K. Dang, J. Shen, L. Dong, Y. Xia, A graph route-based superframe scheduling scheme in wirelesshart mesh networks for high robustness, Wireless Personal Communications 71 (4) (2013) 2431-2444.

[68] N. Sornin, M. Luis, T. Eirich, T. Kramp, O. Hersent, LoRaWAN Specification, Tech. Rep. V1.0.1 Draf 3, LoRa Alliance (October 2015).

URL https://enablingsupport.zendesk.com/hc/en-us/ article_attachments/202357691/LoRaWAN1.0.1_d3.pdf

[69] 01net, Objets connectés : polémique sur la sécurité du réseau français Sigfox, https://www.01net.com/actualites/objets-connectes-lereseau-francais-sigfox-une-passoire-en-matiere-de-securite957875.html, [Online (French); accessed on 21 August 2019] (2019).

[70] S. Andy, B. Rahardjo, B. Hanindhito, Attack scenarios and security analysis of MQTT communication protocol in IoT system, in: International Conference on Electrical Engineering, Computer Science and Informatics (EECSI), IEEE, 2017, pp. 1-6. 
[71] J. Slawomir, Gattacking bluetooth smart devices, Black Hat USA (2016).

[72] W. K. Zegeye, Exploiting bluetooth low energy pairing vulnerability in telemedicine, in: International Telemetering Conference Proceedings, International Foundation for Telemetering, 2015.

[73] X. Fan, F. Susan, W. Long, S. Li, Security analysis of zigbee, Tech. rep., MIT (2017).

URL http://courses.csail.mit.edu/6.857/2017/project/17.pdf

[74] X. Yang, Lorawan: Vulnerability analysis and practical exploitation, Master's thesis, TU Delft. (2017).

URL https://repository.tudelft.nl/islandora/object/uuid: 87730790-6166-4424-9d82-8fe815733f1e?collection=education

[75] X. Yang, E. Karampatzakis, C. Doerr, F. Kuipers, Security vulnerabilities in LoRaWAN, in: International Conference on Internet-of-Things Design and Implementation (IoTDI), IEEE, 2018, pp. 129-140.

[76] A. Reziouk, A. Lebrun, J.-C. Demay, Auditing 6lowpan networks using standard penetration testing tools, DEF CON 24 (2016).

[77] T. Melamed, An active man-in-the-middle attack on bluetooth smart devices, International Journal of Safety and Security Engineering 8 (2) (2018) 200-211.

[78] O. Olawumi, K. Haataja, M. Asikainen, N. Vidgren, P. Toivanen, Three practical attacks against zigbee security: Attack scenario definitions, practical experiments, countermeasures, and lessons learned, in: International Conference on Hybrid Intelligent Systems (HIS), 2014, pp. 199-206.

[79] J. Durech, M. Franekova, Security attacks to ZigBee technology and their practical realization, in: International Symposium on Applied Machine Intelligence and Informatics (SAMI), IEEE, 2014, pp. 345-349.

[80] J. D. Fuller, B. W. Ramsey, Rogue z-wave controllers: A persistent attack channel, in: Local Computer Networks Conference Workshops (LCN), IEEE, 2015, pp. 734-741.

[81] P. Pongle, G. Chavan, A survey: Attacks on RPL and 6LoWPAN in IoT, in: International Conference on Pervasive Computing (ICPC), IEEE, 2015, pp. 1-6.

[82] L. Wallgren, S. Raza, T. Voigt, Routing attacks and countermeasures in the RPLbased internet of things, International Journal of Distributed Sensor Networks (IJDSN) 9 (2013).

[83] G. Lee, J. Lim, D.-k. Kim, S. Yang, M. Yoon, An approach to mitigating sybil attack in wireless networks using zigBee, in: International Conference on Advanced Communication Technology (ICACT), Vol. 2, IEEE, 2008, pp. 10051009. 
[84] M. Mavani, K. Asawa, Modeling and analyses of ip spoofing attack in 6lowpan network, Computers \& Security 70 (2017) 95-110.

[85] P. Perazzo, C. Vallati, D. Varano, G. Anastasi, G. Dini, Implementation of a wormhole attack against a RPL network: Challenges and effects, in: 14th Annual Conference on Wireless On-demand Network Systems and Services (WONS), IEEE, 2018, pp. 95-102.

[86] E. Aras, G. S. Ramachandran, P. Lawrence, D. Hughes, Exploring the security vulnerabilities of LoRa, in: International Conference on Cybernetics (CYBCONF), IEEE, 2017, pp. 1-6.

[87] C. P. O'Flynn, Message denial and alteration on IEEE 802.15. 4 low-power radio networks, in: International Conference on New Technologies, Mobility and Security (NTMS), IEEE, 2011, pp. 1-5.

[88] B. Stelte, G. D. Rodosek, Thwarting attacks on ZigBee-Removal of the KillerBee stinger, in: International Conference on Network and Service Management (CNSM), IEEE, 2013, pp. 219-226.

[89] M. Kurniawan, S. Yazid, Mitigation strategy of sinkhole attack in Wireless Sensor Network, in: International Workshop on Big Data and Information Security (IWBIS), IEEE, 2017, pp. 119-125.

[90] L. Coppolino, V. DAlessandro, S. DAntonio, L. Levy, L. Romano, My smart home is under attack, in: International Conference on Computational Science and Engineering (CSE), IEEE, 2015, pp. 145-151.

[91] L. N. Whitehurst, T. R. Andel, J. T. McDonald, Exploring security in zigbee networks, in: Cyber and Information Security Research Conference (CISR), 2014, pp. 25-28.

[92] D. Cauquil, BtleJuice: the Bluetooth Smart MitM Framework, DEF CON 24 Internet of Things Village, 2016, available at https://www.youtube.com/watch?v=lcn07TclnS0 (accessed on 21 August 2019).

[93] C. L. Schuba, I. V. Krsul, M. G. Kuhn, E. H. Spafford, A. Sundaram, D. Zamboni, Analysis of a denial of service attack on tcp, in: IEEE Symposium on Security and Privacy, IEEE, 1997, pp. 208-223.

[94] S. Sicari, A. Rizzardi, D. Miorandi, A. Coen-Porisini, Reato: Reacting to denial of service attacks in the internet of things, Computer Networks 137 (2018) 3748.

[95] L. Bayou, D. Espes, N. Cuppens-Boulahia, F. Cuppens, Security issue of wirelesshart based SCADA systems, in: International Conference on Risks and Security of Internet and Systems (CRiSIS), Springer, 2015, pp. 225-241. 
[96] J. R. Douceur, The sybil attack, in: International Workshop on Peer-to-Peer Systems (IPTPS), 2002, pp. 251-260.

[97] F. Medjek, D. Tandjaoui, I. Romdhani, N. Djedjig, Performance evaluation of RPL protocol under mobile sybil attacks, in: Trustcom/BigDataSE/ICESS, IEEE, 2017, pp. 1049-1055.

[98] Y.-C. Hu, A. Perrig, D. B. Johnson, Packet leashes: a defense against wormhole attacks in wireless networks, in: Annual Joint Conference of the IEEE Computer and Communications INFOCOM, Vol. 3, IEEE, 2003, pp. 1976-1986.

[99] J. Ding, M. Nemati, C. Ranaweera, J. Choi, Iot connectivity technologies and applications: A survey, IEEE Access 8 (2020) 67646-67673.

[100] S. Tedeschi, C. Emmanouilidis, J. Mehnen, R. Roy, A design approach to iot endpoint security for production machinery monitoring, Sensors 19 (10) (2019) 2355 .

[101] J. Yun, R. C. Teja, N. Chen, N. Sung, J. Kim, Interworking of onem2m-based iot systems and legacy systems for consumer products, in: International Conference on Information and Communication Technology Convergence (ICTC), 2016, pp. 423-428.

[102] E. Baccelli, C. Gündoğan, O. Hahm, P. Kietzmann, M. S. Lenders, H. Petersen, K. Schleiser, T. C. Schmidt, M. Wählisch, Riot: An open source operating system for low-end embedded devices in the iot, IEEE Internet of Things Journal 5 (6) (2018) 4428-4440.

[103] X. Vilajosana, P. Tuset, T. Watteyne, K. Pister, Openmote: Open-source prototyping platform for the industrial iot, in: International Conference on Ad Hoc Networks, Springer, 2015, pp. 211-222.

[104] B. Schneier, The Internet of Things Is Wildly Insecure - And Often Unpatchable, https://www.wired.com/2014/01/theres-no-good-way-topatch-the-internet-of-things-and-thats-a-huge-problem/, [Online; accessed on 24 July 2020] (2014).

[105] F. J. A. Padilla, E. Baccelli, T. Eichinger, K. Schleiser, The future of iot software must be updated, in: IAB Workshop on Internet of Things Software Update (IoTSU), 2016.

[106] T. Walshe, A. Simpson, An empirical study of bug bounty programs, in: IEEE 2nd International Workshop on Intelligent Bug Fixing (IBF), IEEE, 2020, pp. $35-44$.

[107] P. Kearney, Iot security: Experience is an expensive teacher, The Internet of Things: From Data to Insight (2020) 107-120.

[108] M. Bishop, About penetration testing, IEEE Security \& Privacy 5 (6) (2007) 84-87. 
[109] F. Alkhabbas, R. Spalazzese, P. Davidsson, Characterizing internet of things systems through taxonomies: A systematic mapping study, Internet of Things 7 (2019) 100084.

[110] M. Shrestha, C. Johansen, J. Noll, D. Roverso, A methodology for security classification applied to smart grid infrastructures, International Journal of Critical Infrastructure Protection 28 (2020) 100342.

[111] P. Williams, P. Rojas, M. Bayoumi, Security taxonomy in iot - a survey, in: 62nd International Midwest Symposium on Circuits and Systems (MWSCAS), 2019, pp. 560-565.

[112] S. N. Matheu, J. L. Hernandez-Ramos, A. F. Skarmeta, Toward a cybersecurity certification framework for the internet of things, IEEE Security \& Privacy 17 (3) (2019) 66-76.

[113] G. Baldini, A. Skarmeta, E. Fourneret, R. Neisse, B. Legeard, F. Le Gall, Security certification and labelling in internet of things, in: 3rd World Forum on Internet of Things (WF-IoT), 2016, pp. 627-632.

[114] D. A. Robles-Ramirez, P. J. Escamilla-Ambrosio, T. Tryfonas, Iotsec: Uml extension for internet of things systems security modelling, in: International Conference on Mechatronics, Electronics and Automotive Engineering (ICMEAE), IEEE, 2017, pp. 151-156.

[115] S. L. Keoh, S. S. Kumar, H. Tschofenig, Securing the internet of things: A standardization perspective, IEEE Internet of things Journal 1 (3) (2014) 265275.

[116] S. M. M. Fattah, N.-M. Sung, I.-Y. Ahn, M. Ryu, J. Yun, Building iot services for aging in place using standard-based iot platforms and heterogeneous iot products, Sensors 17 (10) (2017) 2311. 